\title{
Synchronizability of Multilayer Star and Star-Ring Networks
}

\author{
Yang Deng, Zhen Jia $\mathbb{D}$, and Feimei Yang \\ College of Science, Guilin University of Technology, Guilin 541004, China \\ Correspondence should be addressed to Zhen Jia; jjjzzz0@163.com
}

Received 25 November 2019; Accepted 17 February 2020; Published 1 April 2020

Academic Editor: Nikos I. Karachalios

Copyright (c) 2020 Yang Deng et al. This is an open access article distributed under the Creative Commons Attribution License, which permits unrestricted use, distribution, and reproduction in any medium, provided the original work is properly cited.

Synchronization of multilayer complex networks is one of the important frontier issues in network science. In this paper, we strictly derived the analytic expressions of the eigenvalue spectrum of multilayer star and star-ring networks and analyzed the synchronizability of these two networks by using the master stability function (MSF) theory. In particular, we investigated the synchronizability of the networks under different interlayer coupling strength, and the relationship between the synchronizability and structural parameters of the networks (i.e., the number of nodes, intralayer and interlayer coupling strengths, and the number of layers) is discussed. Finally, numerical simulations demonstrated the validity of the theoretical results.

\section{Introduction}

Network science is an interdisciplinary subject which abstracts physical, biological, economic and social systems into networks composed of nodes and edges and studies their structural characteristics, dynamic evolution and dynamic characteristics. The network synchronization as an important emerging phenomenon of a population of dynamically interacting units in various fields of science has attracted much attention. Synchronization of complex networks has been widely studied, and much research works have been done over the past few decades and achieved fruitful research results [1-24]. However, most of these works are focusing on isolated networks; in reality, most systems are not isolated but interrelated such as the combination of aviation and railway transportation networks in the transportation system; the interdependence of the server and the terminal system in computer networks; in power infrastructure, the interactive control between the power station and the computer central control system; and in the social network, the compound overlap between the real interpersonal and online interpersonal networks, which constitute more complex networks, called multilayer networks. Therefore, in recent years, the focus of complex network research has gradually shifted from the single layer network to the multilayer network. The research of multilayer network has become an important research direction and attracts tensive attention from scholars.

Although the research on multilayer networks is still in its infancy, a series of influential research results have emerged. In 2013, Gómez et al. proposed a diffusion dynamics model based on multilayer networks and the supraLaplacian matrix of the networks [25]. Granell et al. analyzed the correlation between the two processes of epidemic transmission and epidemic and proposed the information awareness of preventing its infection on the multilayer network [26]. In 2014, Aguirre et al. discussed the eigenvalue spectrum of two completely identical star networks coupled by an edge between layers [27] and considered synchronizability with different coupling modes between layers. In 2016, Xu et al. studied the eigenvalue spectrum and synchronizability of the two-layer star network and theoretically provided the analytic value of the eigenvalue spectrum of the two-layer star network with full connection between layers $[28,29]$. In $2017, \mathrm{Li}$ et al. investigated some rules and properties about synchronizability of duplex networks composed of two networks interconnected by two links, for a specific duplex network composed of two star networks, analytical expressions containing the largest and smallest nonzero eigenvalues of the (weighted) Laplacian matrix, and the interlink weight, as well as the network size, which are 
given for three different interlayer connection patterns [30]. Sun et al. studied the synchronizability of multilayer unidirectional coupling star networks and strictly derived the eigenvalues of such networks in the case of unidirectional coupling between layers [31]. Later, Wei et al. further studied the synchronizability of a double-layer regular network based on the master stability function (MSF) theory by using numerical simulation [32]. In 2019, Tang et al. extended the master stability function to multilayer networks with different intralayer and interlayer coupling functions and derived three master stability equations that determined complete synchronization, intralayer synchronization and interlayer synchronization regions [33]. Deng et al. studied the problem of synchronization of two kinds of multiplex chain networks under different coupling modes between layers and derived the eigenvalue spectrum of the supraLaplacian matrices of those networks [34]. However, due to the structural complexity of multilayer networks, there is almost no strict theoretical derivation on the eigenvalues of the multilayer network; most of the studies are based on the results of numerical simulation on synchronizability of that. To provide more useful foundations for getting insight into understanding synchronizability of multilayer networks and explore the main influencing factors of synchronizability, in our paper, two kinds of typical multilayer networks (i.e., multilayer star and star-ring networks) are considered on the basis of the literature [28] that studied the synchronizability of the two-layer star network; we not only strictly derived the eigenvalue spectrum of the supra-Laplacian matrices of multilayer star and star-ring networks (not limited to two layers) but also studied the relationships between the synchronizability and structural parameters of that.

The paper is structured as follows. Some preliminaries are introduced in Section 2. Section 3 studies the eigenvalue spectrum and synchronizability of the multilayer star networks. In Section 4, the eigenvalue spectrum and synchronizability of the multilayer star-ring networks are studied. Section 5 explores the relationship between the synchronizability and structural parameters of two kinds of multilayer networks. Finally, Section 6 gives the conclusion of this paper.

\section{Preliminaries}

2.1. Dynamics Model of the Multilayer Network. The dynamic equation of the $i$-th node in the multilayer network, with $M$ layers, is $[33,34]$

$$
\begin{aligned}
\dot{x}_{i}^{K}= & f\left(x_{i}^{K}\right)+a \sum_{j=1}^{N} \omega_{i j}^{K} H\left(x_{j}^{K}\right) \\
& +d \sum_{L=1}^{M} d_{i}^{K L} \Gamma\left(x_{i}^{L}\right), \quad i=1,2, \ldots, N ; K=1,2, \ldots, M,
\end{aligned}
$$

where $\dot{x}_{i}^{K}=f\left(x_{i}^{K}\right)(i=1,2, \ldots, N ; K=1,2, \ldots, M)$ describes the isolated dynamics for the $i$-th node in the $K$-th layer, $f(*): \mathbb{R}^{n} \longrightarrow \mathbb{R}^{n}$ is a well-defined vector function, $H(*): \mathbb{R}^{n} \longrightarrow \mathbb{R}^{n}$ and $a$ are the inner coupling function and coupling strength for nodes within each layer, respectively, and $\Gamma(*): \mathbb{R}^{n} \longrightarrow \mathbb{R}^{n}$ and $d$ are the inner coupling function and coupling strength for nodes across layers, respectively. Here, $W^{K}=\left(\omega_{i j}^{K}\right) \in \mathbb{R}^{N \times N}$ is the coupling weight configuration matrix of the $K$-th layer. Explicitly, if the $i$-th node is connected with the $j$-th $(j \neq i)$ node within the $K$-th layer, $\omega_{i j}^{K}=1$; otherwise, $\omega_{i j}^{K}=0$, and there is

$$
\omega_{i i}^{K}=-\sum_{\substack{j=1 \\ j \neq i}}^{N} \omega_{i j}^{K}, \quad i, j=1,2, \ldots, N, K=1,2, \ldots, M .
$$

Let $L^{(K)}=-a W^{K}$, which is a Laplacian matrix. If the $i$-th node in the $K$-th layer is connected with its replica in the $L$-th $(L \neq K)$ layer, $d_{i}^{K L}=1$; otherwise, $d_{i}^{K L}=0$, and there is

$$
d_{i}^{K K}=-\sum_{\substack{L=1 \\ L \neq K}}^{M} d_{i}^{K L}, \quad L, K=1,2, \ldots, M .
$$

It is obvious that $D=\left(d_{i}^{K L}\right) \in \mathbb{R}^{M \times M}$ is also a negative Laplacian matrix.

Let $\mathscr{L}$ be the supra-Laplacian matrix of equation (1), $\mathscr{L}_{\mathrm{I}}$ be the supra-Laplacian matrix representing the interlayer topology, and $\mathscr{L}_{L}$ be the supra-Laplacian matrix describing the intralayer topology. Then, $\mathscr{L}$ can be written as

$$
\mathscr{L}=\mathscr{L}_{I}+\mathscr{L}_{L}
$$

Taking $L_{\mathrm{I}}$ to be the Laplacian matrix of the interlayer networks, we have

$$
\mathscr{L}_{I}=L_{I} \otimes I_{N},
$$

where $\otimes$ is the Kronecker product, $L_{\mathrm{I}}=-d D$, and $I_{N}$ is the $N$ $\times N$ identity matrix. As for $\mathscr{L}_{L}$, it can be represented by the direct sum of the Laplacian matrix $L^{(K)}$ within each layer, namely,

$$
\mathscr{L}_{L}=\left(\begin{array}{cccc}
L^{(1)} & 0 & \cdots & 0 \\
0 & L^{(2)} & \cdots & 0 \\
\vdots & \vdots & & \vdots \\
0 & 0 & \cdots & L^{(M)}
\end{array}\right)=\underset{K=1}{M} L^{(K)} .
$$

The eigenvalues of the supra-Laplacian matrix of the networks are recorded as $0=\lambda_{1}<\lambda_{2} \leq \lambda_{3} \leq \cdots \leq \lambda_{\text {max }}$. According to the MSF theory, the synchronizability of network (1) is determined by the minimum nonzero eigenvalue or the ratio $R=\lambda_{\max } / \lambda_{2}$ of the maximum eigenvalue to the minimum nonzero eigenvalue of the supra-Laplacian matrix $\mathscr{L}$. Generally, when the network synchronous region is unbounded, the greater the $\lambda_{2}$, the stronger the synchronizability is; when the synchronous region is bounded, the smaller the $R=\lambda_{\max } / \lambda_{2}$, the stronger the synchronizability is.

2.2. Two Types of Multilayer Networks. This paper focuses on two types of multilayer networks, that is, the multilayer star and star-ring networks. For a multilayer star network, each layer is made up of identical star subnets, as shown in Figure 1(a), a three-layer star network. For a multilayer 


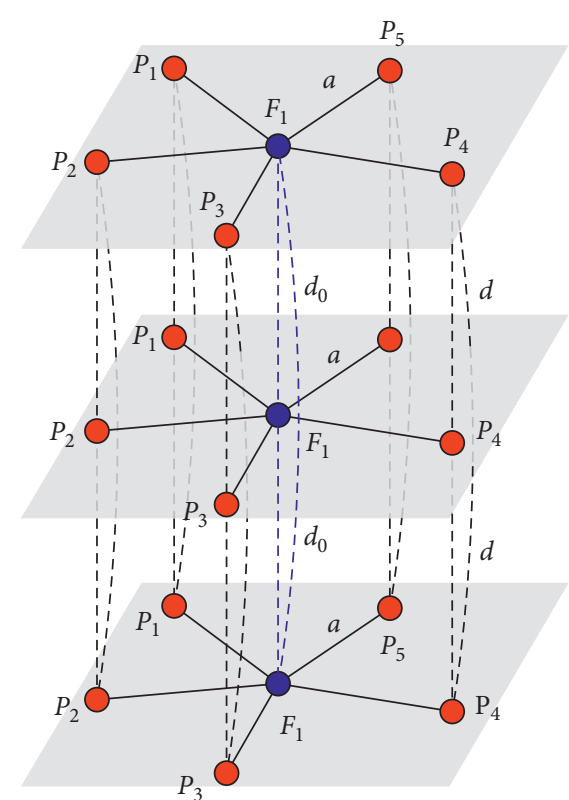

(a)

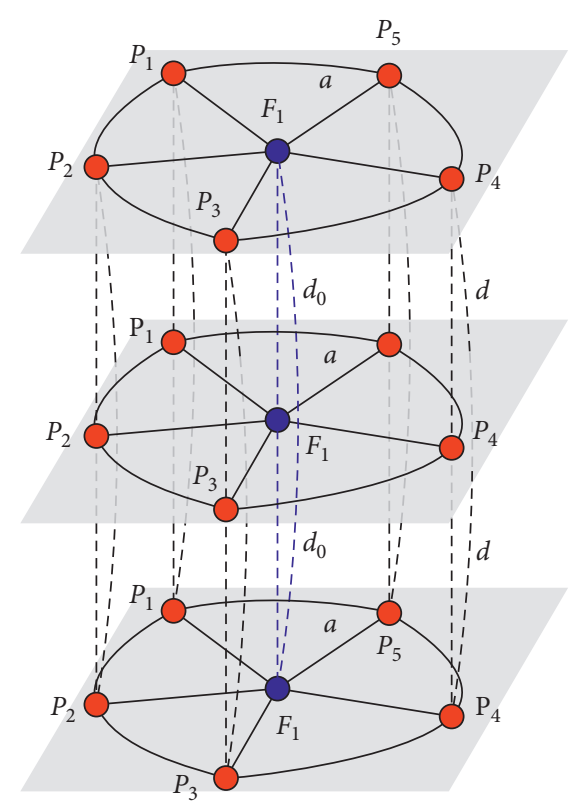

(b)

FIgURE 1: Schematic diagram of the network structure. (a) A three-layer star network structure. (b) A three-layer star-ring network structure.

star-ring network, each layer is made up of identical starring subnets, as shown in Figure 1(b), a three-layer starring network. The nodes in each layers carry the identical dynamics, and each node in one layer is connected to its replicas in other layers (the hub node $F_{1}$ is connected to its replicas in other layers, and the leaf nodes $P_{i}$ are connected to their replicas in other layers). Suppose each multilayer network has $M$ layers and $N * M$ nodes (each layer contains $N$ nodes), the intralayer coupling strength of each layer is $a$, and the interlayer coupling strength between the hub nodes is $d_{0}$ and between the leaf nodes is $d$.

In order to analyze the synchronizability of these two types of multilayer networks, we will separately calculate the eigenvalue spectrum of the networks and analyze the relationship between the synchronizability and structural parameters in the following sections.

\section{The Eigenvalue Spectrum and Synchronizability of Multilayer Star Networks}

For the derivation of the network eigenvalues, let us introduce the following lemma.
Lemma 1 (see [31]). Let $A, B$ be $N \times N$ matrices and $M$ be an integer, then

$$
\left|\begin{array}{cccc}
A & B & \cdots & B \\
B & A & \cdots & B \\
\vdots & \vdots & \ddots & \vdots \\
B & B & \cdots & A
\end{array}\right|_{M \times M}=|A+(M-1) B| \cdot|A-B|^{(M-1)}
$$

Considering the star networks composed of $M$ layers (each layer consisting of $N$ nodes), the node dynamics equation of the networks is as shown in equation (1). In the following, we derive the eigenvalue spectrum and synchronizability of the multilayer star networks under two different conditions, namely, the interlayer coupling strength between the hub nodes $d_{0}$ and between the leaf nodes $d$ is different $\left(d_{0} \neq d\right)$ or the same $\left(d_{0}=d\right)$.

3.1. The Eigenvalue Spectrum and Synchronizability of Multilayer Star Networks with $d_{0} \neq d$. When $d_{0} \neq d$, the supraLaplacian matrix of the multilayer star networks is 


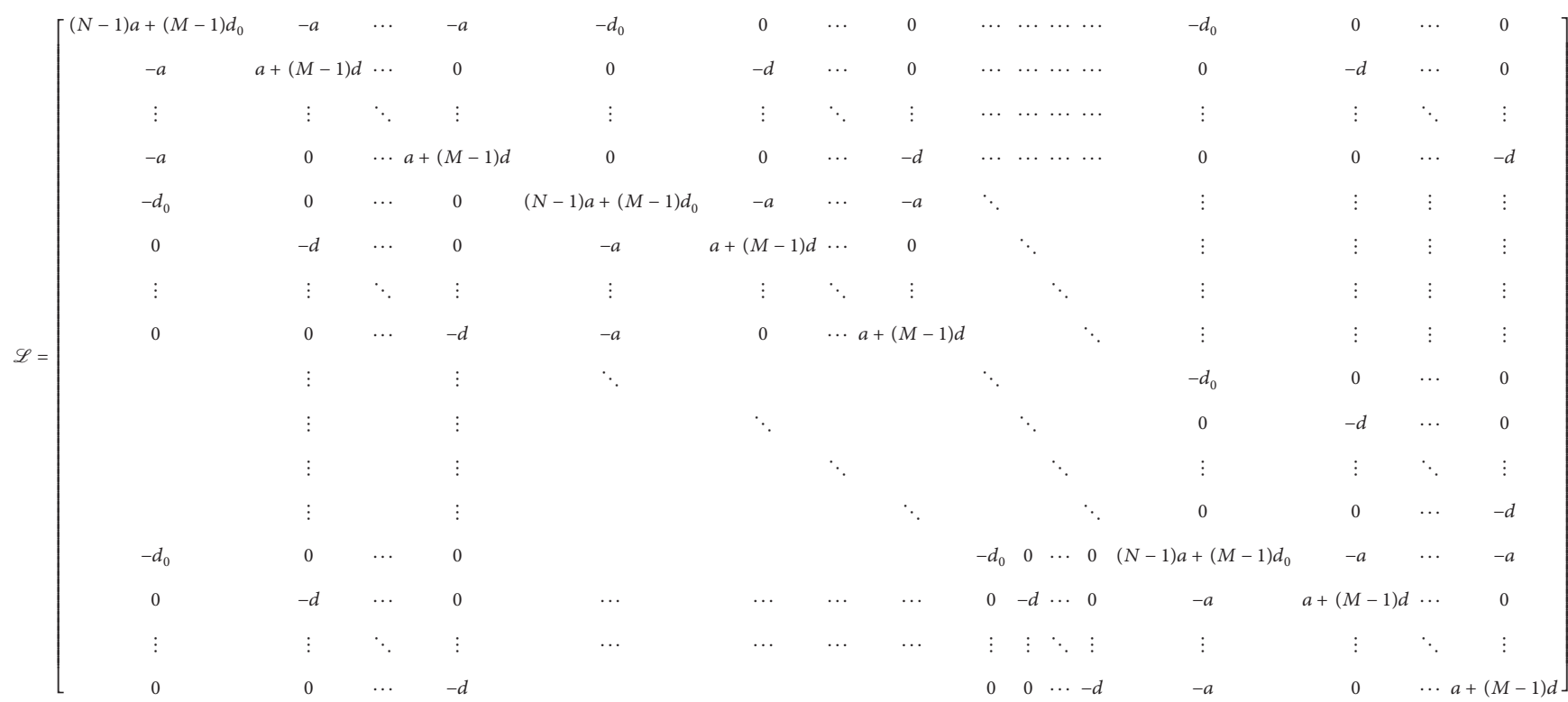

According to Lemma 1, one obtains the characteristic polynomial of matrix $\mathscr{L}$ as

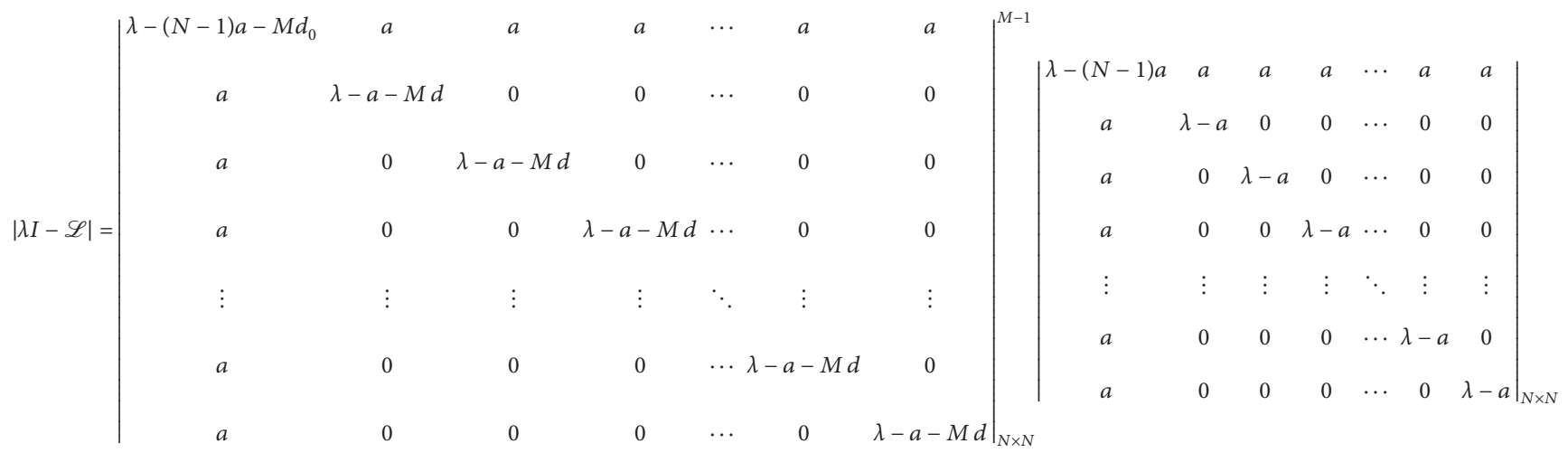

$$
\begin{aligned}
& =\lambda(\lambda-N a)(\lambda-a)^{N-2}(\lambda-a-M d)^{(N-2)(M-1)}\left(\lambda-\frac{N a+M d+M d_{0}+\sqrt{\left(N a+M d+M d_{0}\right)^{2}-4\left[(N-1) a M d+a M d_{0}+M^{2} d d_{0}\right]}}{2}\right)^{M-1} \\
& \cdot\left(\lambda-\frac{N a+M d+M d_{0}-\sqrt{\left(N a+M d+M d_{0}\right)^{2}-4\left[(N-1) a M d+a M d_{0}+M^{2} d d_{0}\right]}}{2}\right)^{M-1} .
\end{aligned}
$$

$$
\begin{array}{lr}
\text { Let } & \lambda_{\mathrm{I}}=\left(\left(N a+M d+M d_{0}\right)\right. \\
\left.\sqrt{\left(N a+M d+M d_{0}\right)^{2}-4\left[(N-1) a M d+a M d_{0}+M^{2} d d_{0}\right]}\right) / 2 \\
\text { and } \quad \lambda_{\mathrm{II}}=\left(\left(N a+M d+M d_{0}\right)+\right. \\
\left.\sqrt{\left(N a+M d+M d_{0}\right)^{2}-4\left[(N-1) a M d+a M d_{0}+M^{2} d d_{0}\right]}\right) / 2
\end{array}
$$

Therefore, the eigenvalues of $\mathscr{L}$ are $0, N a, \underbrace{a \cdots a}_{N-2}$, $\underbrace{a+M d \cdots a+M d}_{(N-2)(M-1)}, \underbrace{\lambda_{\mathrm{I}} \cdots \lambda_{\mathrm{I}}}_{M-1}, \underbrace{\lambda_{\mathrm{II}} \cdots \lambda_{\mathrm{II}}}_{M-1}$, where $a$ is the $N-2$ multiple roots, $a+M d$ is the $(N-2)(M-1)$ multiple roots, 
and $\lambda_{\mathrm{I}}$ and $\lambda_{\mathrm{II}}$ are the $M-1$ multiple roots. Through simplification, one gets

$$
\begin{aligned}
\lambda_{\mathrm{I}} & =\frac{N a+M d+M d_{0}-\sqrt{\left(N a+M d+M d_{0}\right)^{2}-4\left[(N-1) a M d+a M d_{0}+M^{2} d d_{0}\right]}}{2} \\
& =\frac{N a+M d+M d_{0}-\sqrt{\left(N a+M d_{0}-M d\right)^{2}+4 M a\left(d-d_{0}\right)}}{2} \\
& =\frac{1}{2} N a\left[1+\frac{M d+M d_{0}}{N a}-\sqrt{\left.\left(1+\frac{M d_{0}-M d}{N a}\right)^{2}+\frac{4 M a\left(d-d_{0}\right)}{(N a)^{2}}\right], \quad N \gg a, d, d_{0}, M}\right. \\
& \approx \frac{1}{2} N a\left[1+\frac{M d+M d_{0}}{N a}-\sqrt{\left(1+\frac{M d_{0}-M d}{N a}\right)^{2}}\right], \quad N \gg a, d, d_{0}, M \\
& =M d .
\end{aligned}
$$

Therefore, for sufficiently large $N$, there is $\lambda_{\mathrm{I}} \longrightarrow M d$. In a similar way, there is

$$
\lambda_{\mathrm{II}}=\frac{N a+M d+M d_{0}+\sqrt{\left(N a+M d+M d_{0}\right)^{2}-4\left[(N-1) a M d+a M d_{0}+M^{2} d d_{0}\right]}}{2} \longrightarrow N a+M d_{0}, \quad N \gg a, d, d_{0}, M .
$$

For sufficiently large $N$, there are $\lambda_{2}=\min \left\{\lambda_{\mathrm{I}}, a\right\} \longrightarrow$ $\min \{M d, a\}$ and $R=\left(\lambda_{\max } / \lambda_{2}\right)=\left(\lambda_{\mathrm{II}} / \min \left\{\lambda_{I}, a\right\}\right) \longrightarrow$ $\left(\left(N a+M d_{0}\right) / \min \{M d, a\}\right)$. To begin with, according to the MSF theory, we study synchronizability of multilayer star networks with different interlayer coupling strength $\left(d_{0} \neq d\right)$, as shown in Table 1.

3.2. The Eigenvalue Spectrum and Synchronizability of the Multilayer Star Networks with $d_{0}=d$. When $d_{0}=d$, one gets the characteristic polynomial of the supra-Laplacian matrix $\mathscr{L}$ :

$$
\begin{aligned}
|\lambda I-\mathscr{L}|= & \lambda(\lambda-N a)(\lambda-a)^{N-2}(\lambda-a-M d)^{(N-2)(M-1)} \\
& (\lambda-N a-M d)^{(M-1)}(\lambda-M d)^{(M-1)}
\end{aligned}
$$

The eigenvalues of the networks are $0, N a, \underbrace{a \cdots a}$, $\underbrace{a+M d \cdots a+M d}, \quad \underline{N a+M d \cdots N a+M d}, \underbrace{M d \cdots M d}$. $(N-2)(M-1)$ $M-1$ According to the MSF theory, $\lambda_{2}=\min \{M d, a\}$ and $R=\lambda_{\max } / \lambda_{2}=((N a+M d) / \min \{M d, a\})$. Then, we study synchronizability of multilayer star networks with invariant interlayer coupling strength $\left(d_{0}=d\right)$, as shown in Table 2 .

It is worth noting that, when $M=2$, the results about the synchronizability varying with structural parameters of multilayer star networks are basically consistent with the literature [28], but our results are more general. According to Tables 1 and 2, for sufficiently large $N$ and $d_{0} \neq d$, when the synchronous region is unbounded, the synchronizability of the multilayer star networks depends on the smaller of $a$ and $M d$, when $a>M d$, the synchronizability is determined by $M d$, and when $a<M d$, the synchronizability is determined by $a$. Therefore, the synchronizability of the networks is not affected by the number of nodes $N$. For bounded synchronous region, the synchronizability depends on $\mathrm{N}+$ $\left(M d_{0} / a\right)$ or $\left(N a+M d_{0}\right) / M d$. When $a<M d$, the synchronizability is determined by $N+\left(M d_{0} / a\right)$, and the synchronizability is weakened with the increase of $N, M$, and $d_{0}$ and strengthened with the increase of $a$. When $a>M d$, the synchronizability of the networks is determined by $\mathrm{Na}+M d_{0} / M d$, and the synchronizability of the network is 
TABLE 1: When $d_{0} \neq d$, the synchronizability of the multilayer star networks varies with $N, a, d, d_{0}$, and $M$.

\begin{tabular}{|c|c|c|c|c|}
\hline & \multicolumn{4}{|c|}{ The multilayer star networks } \\
\hline & \multicolumn{2}{|c|}{$\begin{array}{l}\text { The case of synchronous } \\
\text { region unbounded }\end{array}$} & \multicolumn{2}{|c|}{$\begin{array}{l}\text { The case of } \\
\text { synchronous } \\
\text { region } \\
\text { bounded }\end{array}$} \\
\hline & $\begin{array}{l}a< \\
M d\end{array}$ & $a>M d$ & $\begin{array}{l}a< \\
M d\end{array}$ & $\begin{array}{l}a> \\
M d\end{array}$ \\
\hline & \multicolumn{4}{|c|}{ Synchronizability } \\
\hline $\begin{array}{l}\text { With the } \\
\text { increase of } N\end{array}$ & - & $\begin{array}{c}\text { - }(N \text { is sufficiently } \\
\text { large })\end{array}$ & $\downarrow$ & $\downarrow$ \\
\hline $\begin{array}{l}\text { With the increase } \\
\text { of } M\end{array}$ & - & 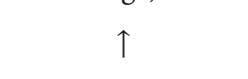 & $\downarrow$ & $\uparrow$ \\
\hline $\begin{array}{l}\text { With the increase } \\
\text { of } a\end{array}$ & $\uparrow$ & - & $\uparrow$ & $\downarrow$ \\
\hline $\begin{array}{l}\text { With the increase } \\
\text { of } d\end{array}$ & - & $\uparrow$ & - & $\uparrow$ \\
\hline $\begin{array}{l}\text { With the increase } \\
\text { of } d_{0}\end{array}$ & - & - & $\downarrow$ & $\downarrow$ \\
\hline
\end{tabular}

-: unchange; $\uparrow$ : strengthen; $\downarrow$ : weaken.

strengthened with the increase of $M$ and $d$ and weakened with the increase of $a, N$, and $d_{0}$; hence, $d$ and $a$ have the opposite effect; when $N$ is fixed and $d$ is increased, the synchronizability of the networks can be maintained invariant by increasing $a$. For $d_{0}=d$, the synchronizability varying with structural parameters is basically consistent with that under the different interlayer coupling strength; the difference is that, when $a<M d$, the synchronizability with invariant interlayer coupling strength is weakened with increasing $d$, while that
TABLE 2: When $d_{0}=d$, the synchronizability of the multilayer star networks varies with $N, a, d$, and $M$.

\begin{tabular}{|c|c|c|c|c|}
\hline & \multicolumn{4}{|c|}{ The multilayer star networks } \\
\hline & $\begin{array}{l}\text { The } \\
\text { synch } \\
\text { re } \\
\text { unbo }\end{array}$ & $\begin{array}{l}\text { of } \\
\text { ous } \\
\text { led }\end{array}$ & $\begin{array}{l}\text { The } \\
\text { synch } \\
\text { region }\end{array}$ & $\begin{array}{l}\text { ase of } \\
\text { onous } \\
\text { ounded }\end{array}$ \\
\hline & $a<M d$ & $>M d$ & $a<M d$ & $a>M d$ \\
\hline & \multicolumn{4}{|c|}{ Synchronizability } \\
\hline With the increase of $N$ & - & - & $\downarrow$ & $\downarrow$ \\
\hline With the increase of $M$ & - & $\uparrow$ & $\downarrow$ & $\uparrow$ \\
\hline With the increase of $a$ & $\uparrow$ & - & $\uparrow$ & $\downarrow$ \\
\hline With the increase of $d$ & - & $\uparrow$ & $\downarrow$ & $\uparrow$ \\
\hline
\end{tabular}

with different interlayer coupling strength is invariant with increasing $d$, with the bounded synchronous region.

\section{The Eigenvalue Spectrum and Synchronizability of Multilayer Star- Ring Networks}

In this section, we explore the eigenvalue spectrum and synchronizability of the multilayer star-ring networks in the case of $d_{0} \neq d$ and $d_{0}=d$, respectively.

4.1. The Eigenvalue Spectrum and Synchronizability of Multilayer Star-Ring Networks with $d_{0} \neq d$. For multilayer starring networks, when $d_{0} \neq d$, one obtains the supra-Laplacian matrix $\mathscr{L}$ of the networks:

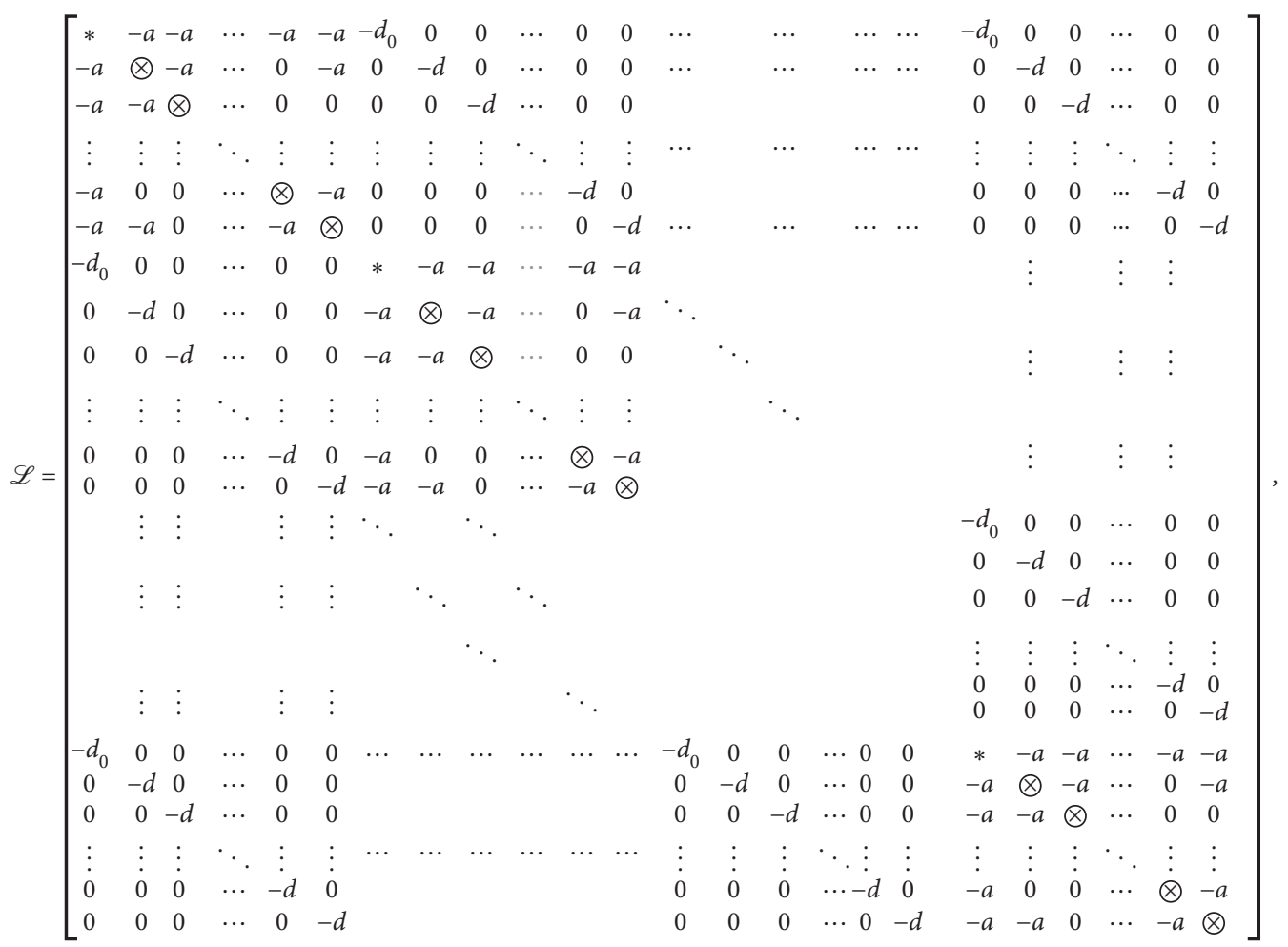


where $* \hat{=}(N-1) a+(M-1) d_{0}$ and $\otimes \hat{=} 3 a+(M-1) d$.

According to Lemma 1 , the characteristic polynomial of $\mathscr{L}$ is

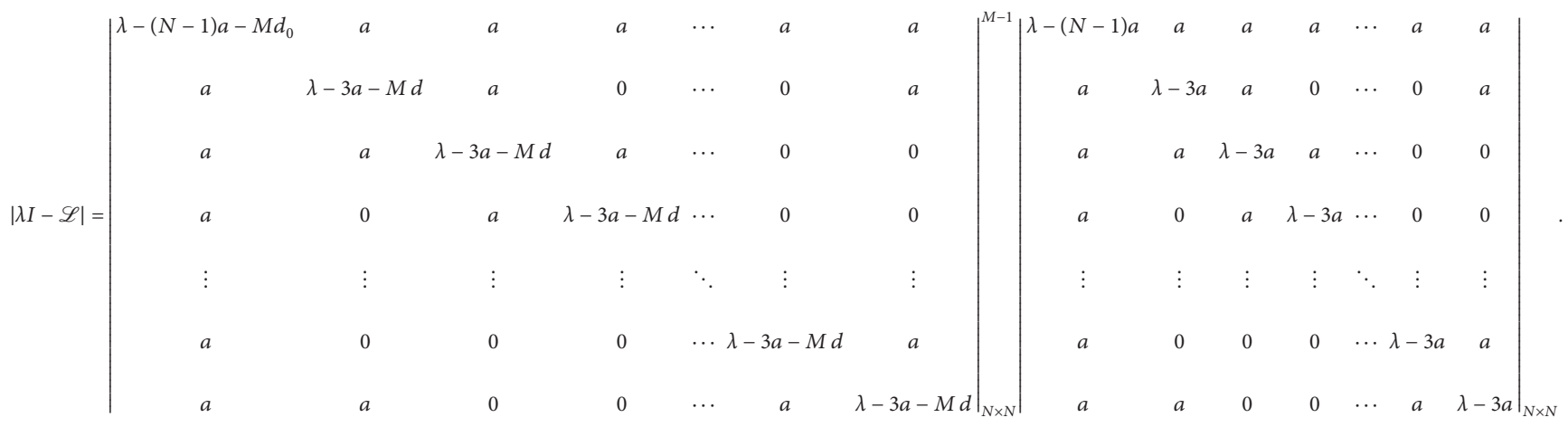

Let

$$
\begin{aligned}
& |C|_{N \times N}^{M-1}=\left(\left.\begin{array}{ccccccc}
\lambda-(N-1) a-M d_{0} & a & a & a & \cdots & a & a \\
a & \lambda-3 a-M d & a & 0 & \cdots & 0 & a \\
a & a & \lambda-3 a-M d & a & \cdots & 0 & 0 \\
a & 0 & a & \lambda-3 a-M d & \ldots & 0 & 0 \\
\vdots & \vdots & \vdots & \vdots & \ddots & \vdots & a \\
a & 0 & 0 & 0 & \cdots & \lambda-3 a-M d & a \\
& a & 0 & 0 & \cdots & a & \lambda-3 a-M d
\end{array}\right|_{N \times N} ^{M-1}\right. \\
& |D|_{N \times N}=\left|\begin{array}{ccccccc}
\lambda-(N-1) a & a & a & a & \cdots & a & a \\
a & \lambda-3 a & a & 0 & \cdots & 0 & a \\
a & a & \lambda-3 a & a & \cdots & 0 & 0 \\
a & 0 & a & \lambda-3 a & \cdots & 0 & 0 \\
\vdots & \vdots & \vdots & \vdots & \ddots & \vdots & \vdots \\
a & 0 & 0 & 0 & \cdots & \lambda-3 a & a \\
a & a & 0 & 0 & \cdots & a & \lambda-3 a
\end{array}\right|_{N \times N}
\end{aligned}
$$

By simplifying,

$$
\begin{aligned}
|C|_{n}^{M-1}= & \left(\lambda-\frac{N a+M d+M d_{0}+\sqrt{\left(N a+M d+M d_{0}\right)^{2}-4\left[(N-1) a M d+a M d_{0}+M^{2} d d_{0}\right]}}{2}\right)^{M-1} \\
& \cdot\left(\lambda-\frac{N a+M d+M d_{0}-\sqrt{\left(N a+M d+M d_{0}\right)^{2}-4\left[(N-1) a M d+a M d_{0}+M^{2} d d_{0}\right]}}{2}\right)^{M-1}|H|_{(N-1) \times(N-1)}^{M-1},
\end{aligned}
$$


where

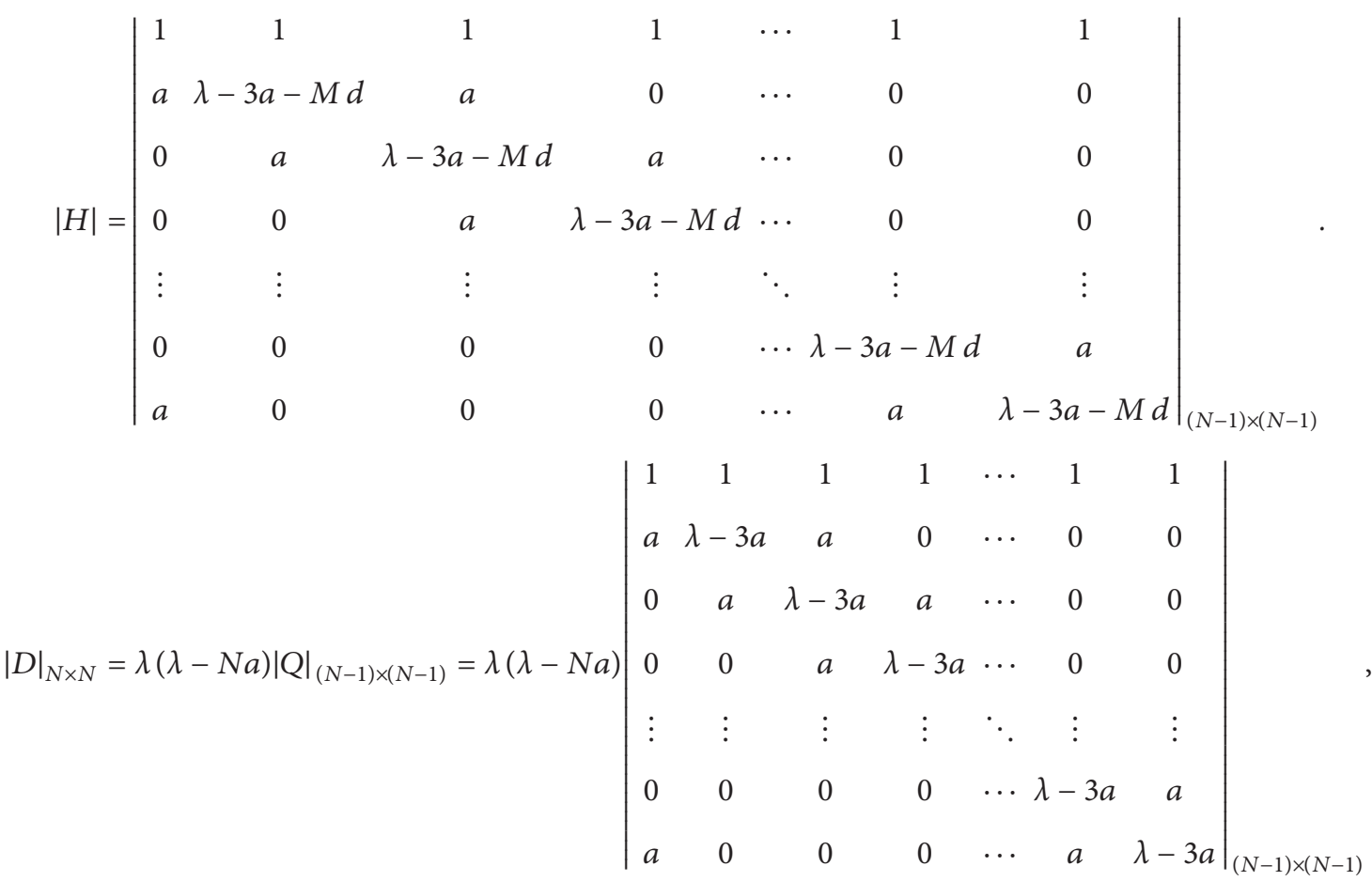

When $N$ is an odd number, the values of $\lambda$ in $|Q|$ are $5 a, \underbrace{a+4 a \sin ^{2}((k \pi) /(2(N-1)))}_{2}, \quad k=2,4,6, \ldots, N-3$.

When $N$ is an even number, the values of $\lambda$ in $|Q|$ are $\underbrace{a+4 a \sin ^{2}((k \pi) /(2(N-1)))}_{2}, \quad k=2,4,6, \ldots, N-2$.

When $N$ is an odd number, the values of $\lambda$ in $|H|$ are
$M d+5 a, \underbrace{M d+a+4 a \sin ^{2}((k \pi) /(2(N-1)))}$,

$$
k=2,4,6, \ldots, N-3 \text {. }
$$

When $N$ is an even number, the values of $\lambda$ in $|H|$ are $\underbrace{a+4 a \sin ^{2}((k \pi) /(2(N-1)))}_{2}, \quad k=2,4,6, \ldots, N-2$.

Thus, the eigenvalues of $\mathscr{L}$ are as follows: When $N$ is an odd number:

$0,5 a, N a, \underbrace{a+4 a \sin ^{2}\left(\frac{k \pi}{2(N-1)}\right)}_{2}, \quad(k=2,4,6, \ldots, N-3), \underbrace{M d+5 a}_{M-1}, \underbrace{M d+a+4 a \sin ^{2}\left(\frac{k \pi}{2(N-1)}\right)}_{2(M-1)}, \quad(k=2,4,6, \ldots, N-3)$

$\underbrace{\frac{N a+M d+M d_{0}-\sqrt{\left(N a+M d+M d_{0}\right)^{2}-4\left[(N-1) a M d+a M d_{0}+M^{2} d d_{0}\right]}}{2}}_{M-1}$,

$\underbrace{\frac{N a+M d+M d_{0}+\sqrt{\left(N a+M d+M d_{0}\right)^{2}-4\left[(N-1) a M d+a M d_{0}+M^{2} d d_{0}\right]}}{2}}_{M-1}$. 
TABLE 3: When $d_{0} \neq d$, the synchronizability of the multilayer star-ring networks varies with $N, a, d, d_{0}$, and $M$.

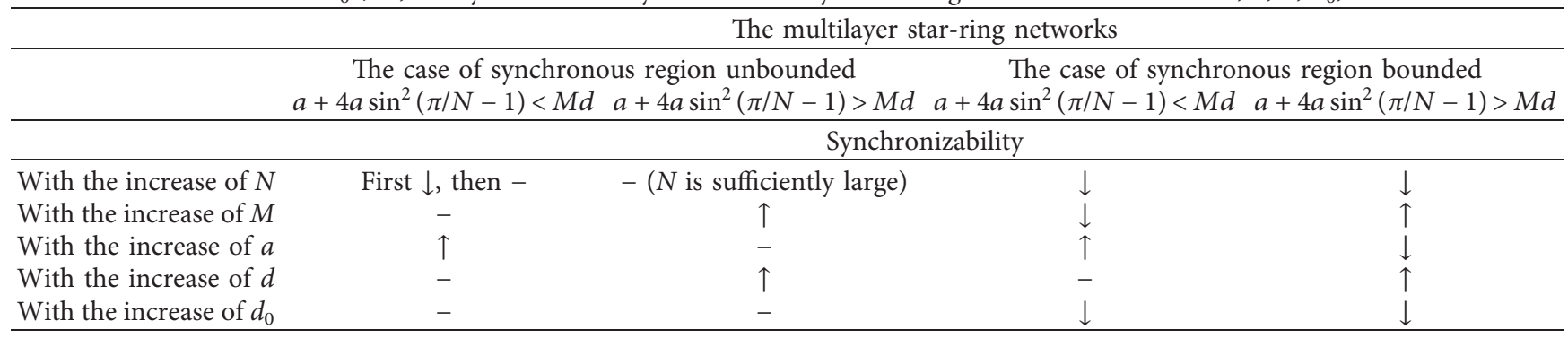

1-: unchange; $\uparrow$ : strengthen; $\downarrow$ : weaken.

When $N$ is an even number:

$$
\begin{aligned}
& \underbrace{0, N a, \underbrace{a+4 a \sin ^{2}\left(\frac{k \pi}{2(N-1)}\right)}, \quad k=2,4,6, \ldots, N-2,}_{2} \\
& \underbrace{M d+a+4 a \sin ^{2}\left(\frac{k \pi}{2(N-1)}\right)}_{2(M-1)}, \quad k=2,4,6, \ldots, N-2, \\
& \underbrace{N a+M d+M d_{0}-\sqrt{\left(N a+M d+M d_{0}\right)^{2}-4\left[(N-1) a M d+a M d_{0}+M^{2} d d_{0}\right]}}_{M-1},
\end{aligned}
$$

$$
\underbrace{\frac{N a+M d+M d_{0}+\sqrt{\left(N a+M d+M d_{0}\right)^{2}-4\left[(N-1) a M d+a M d_{0}+M^{2} d d_{0}\right]}}{2}}_{M-1} .
$$

Let $\quad \lambda_{\text {III }}=N a+M d+M d_{0}-$ $\sqrt{\left(N a+M d+M d_{0}\right)^{2}-4\left[(N-1) a M d+a M d_{0}+M^{2} d d_{0}\right]} / 2$ and $\quad \lambda_{\mathrm{IV}}=\mathrm{Na}+\mathrm{Md}+\mathrm{Md} d_{0}+$ $\sqrt{\left(N a+M d+M d_{0}\right)^{2}-4\left[(N-1) a M d+a M d_{0}+M^{2} d d_{0}\right]} / 2$. Whether $N$ is an odd or even number, $\lambda \max =\lambda_{\mathrm{IV}}$ and $\lambda_{2}=\min \left\{\lambda_{\text {III }}, a+4 a \sin ^{2}(\pi /(N-1))\right\}$. Similar to Section 3.1 , for sufficiently large $N, \lambda_{\mathrm{III}} \longrightarrow M d$ and $\lambda_{\mathrm{IV}} \longrightarrow N a+$ $M d_{0}$; therefore, $\lambda_{2}=\min \left\{\lambda_{\mathrm{III}}, a+4 a \sin ^{2}(\pi / N-1)\right\} \longrightarrow$ $\min \left\{M d, a+4 a \sin ^{2}(\pi / N-1)\right\}$ and $R=\lambda_{\max } / \lambda_{2}\left(\lambda_{\mathrm{IV}} / \min \right.$ $\left.\left\{\lambda_{\mathrm{III}}, a+4 a \sin ^{2}(\pi / N-1)\right\}\right) \longrightarrow\left(N a+M d_{0}\right) / \min \{M d, a+$ $\left.4 a \sin ^{2}(\pi / N-1)\right\}$. According to the MSF theory, we can summarize the relationship between the synchronizability and structural parameters under the different interlayer coupling strength, as shown in Table 3.

4.2. The Eigenvalue Spectrum and Synchronizability of Multilayer Star-Ring Networks with $d_{0}=d$. Similarly, when $d_{0}=$ $d$, the eigenvalues of $\mathscr{L}$ are as follows:

When $N$ is an odd number: $0,5 a, N a$, $\underbrace{a+4 a \sin ^{2}(k \pi / 2(N-1))}_{2},(k=2,4,6, \ldots, N-3), \quad \underbrace{M d}_{M-1}$, $\underbrace{M d+5 a}_{M-1}, \underbrace{M d+a+4 a \sin ^{2}(k \pi / 2(N-1))}_{2(M-1)},(k=2,4,6, \ldots$ $\ldots, N-3), \underbrace{N a+M d}$. When $N$ is an even number: $0, N a$, $\underbrace{a+4 a \sin ^{2}(k \pi / 2(N-1))}_{2},(k=2,4,6, \ldots, N-2), \quad \underbrace{M d}_{M-1}$, $\underbrace{M d+a+4 a \sin ^{2}(k \pi / 2(N-1))},(k=2,4,6, \ldots, N \quad-2)$, $\underbrace{N a+M d}_{M-1}$. Hence, $\lambda \max =N a+M d$ and $\lambda_{2}=\min$ $\left\{M d, a+4 a \sin ^{2}(\pi / N-1)\right\}$. According to the MSF theory, we study synchronizability of multilayer star-ring networks with invariant interlayer coupling strength $\left(d_{0}=d\right)$, as shown in Table 4.

The synchronizability of two kinds of multilayer networks varying with the structural parameters under different $\left(d_{0} \neq d\right)$ interlayer coupling strength is basically the same as that under the same $\left(d_{0}=d\right)$ interlayer coupling strength.

\section{Numerical Simulation}

In this section, we will investigate the synchronizability of multilayer star and star-ring networks under different interlayer coupling strength $\left(d_{0}>d, d_{0}<d\right.$, and $\left.d_{0}=d\right)$ through a large number of numerical simulation experiments.

5.1. The Synchronizability of Multilayer Star Networks. For different interlayer coupling strength (i.e., $d_{0}>d, d_{0}<d$, and $d_{0}=d$.), the synchronizability of multilayer star networks varying with the structural parameters is shown in Figures 2-8. 
TABLE 4: When $d_{0}=d$, the synchronizability of the multilayer star-ring networks varies with $N, a, d$, and $M$.

\begin{tabular}{|c|c|c|c|c|}
\hline & \multicolumn{4}{|c|}{ The multilayer star-ring networks } \\
\hline & $\begin{array}{l}\text { The case of sync } \\
a+4 a \sin ^{2}(\pi / N-1)\end{array}$ & $\begin{array}{l}\text { lbounded } \\
\pi / N-1)>M d\end{array}$ & $\begin{array}{r}\text { The case } \\
a+4 a \sin ^{2}(\pi / 1\end{array}$ & $\begin{array}{l}\text { sounded } \\
\pi / N-1)>M d\end{array}$ \\
\hline & \multicolumn{4}{|c|}{ Synchronizability } \\
\hline With the increase of $N$ & First $\downarrow$, then - & - & $\downarrow$ & $\downarrow$ \\
\hline With the increase of $M$ & - & $\uparrow$ & $\downarrow$ & $\uparrow$ \\
\hline With the increase of $a$ & $\uparrow$ & - & $\uparrow$ & $\downarrow$ \\
\hline With the increase of $d$ & - & $\uparrow$ & $\downarrow$ & $\uparrow$ \\
\hline
\end{tabular}

1-: unchange; $\uparrow$ : strengthen; $\downarrow$ : weaken.

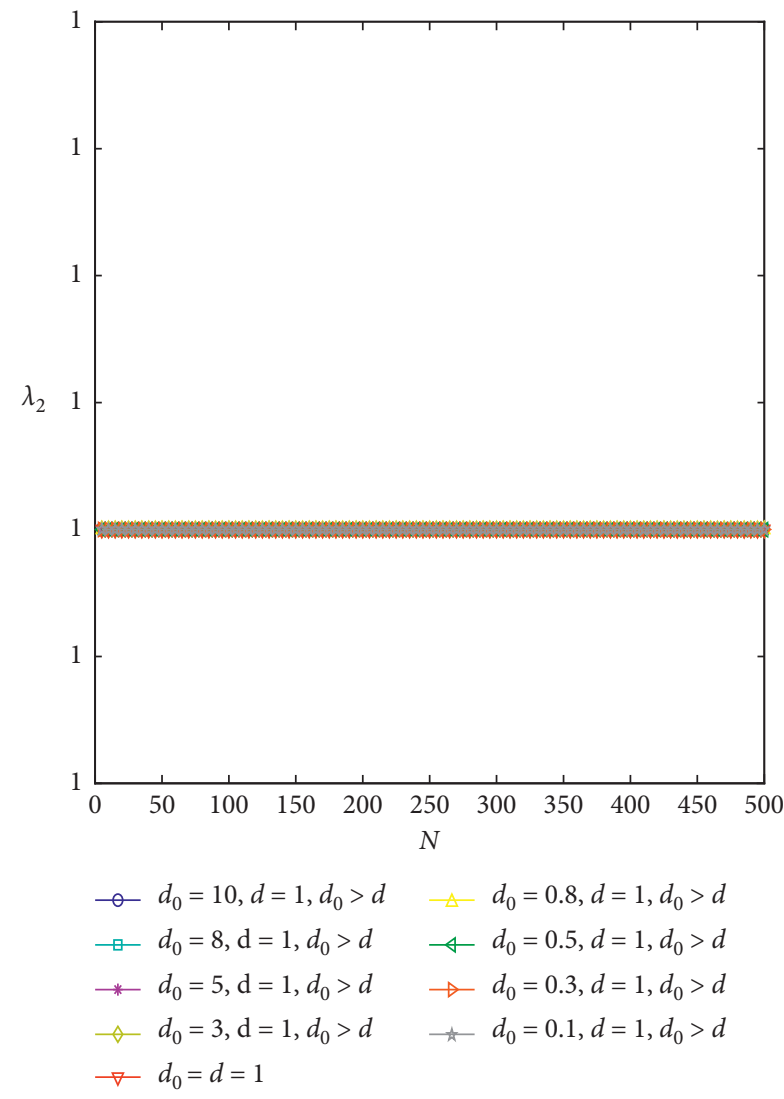

(a)

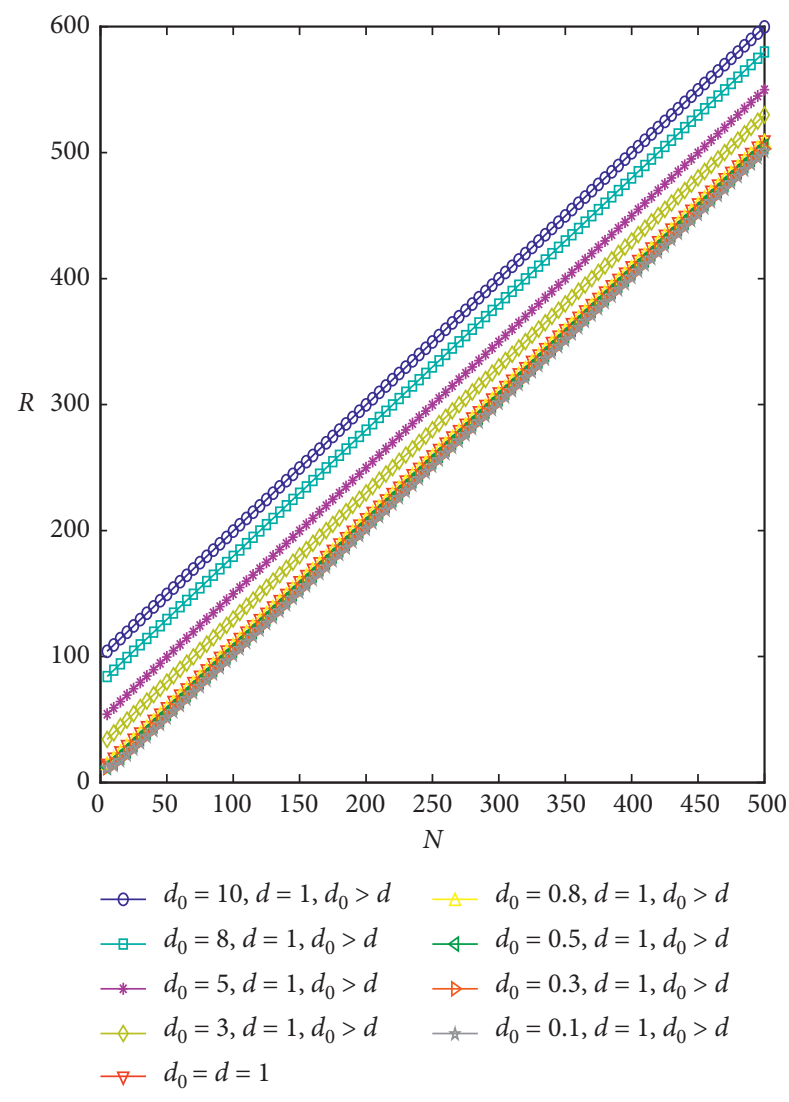

(b)

FIGURE 2: The synchronizability of multilayer star networks $v s$. varying the number of nodes $N(a=1, M=10, d=1$, and $a<M d)$. (a) $\lambda_{2}$ with respect to varying $N$ for different $d_{0}$. (b) $R$ with respect to varying $N$ for different $d_{0}$.

When $a<M d$, it can be seen from the left panel of Figure 2 that $\lambda_{2}$ remains invariant at a specific value $\lambda_{2}=a=$ 1 with increasing $N$ for different $d_{0}$ and different values of $d_{0}$ have no effect on the synchronizability of the networks, with the unbounded synchronous region. The right panel reveals that $R$ increases with increasing $N$, and the observation reveals that the synchronizability is weakened with increasing the number of nodes in each layer. The smaller $d_{0}$, the stronger the synchronizability, with the bounded synchronous region. When $a>M d$, from Figure 3(a), $\lambda_{2}$ remains invariant at a specific value $\lambda_{2}=M d=10$ with increasing $N$ for $d_{0}=d ; \lambda_{2}$ increases at first and then almost levels off at an upper bound value $\lambda_{2}=M d=10$ for $d_{0}<d ; \lambda_{2}$ decreases at first and then almost levels off at a lower bound value $\lambda_{2}=M d=10$ for $d_{0}>d$, with the unbounded synchronous region. From Figure 3(b), $R$ increases with increasing $N$, the synchronizability is weakened with increasing $N$, and different values of $d_{0}$ have no effect on the synchronizability of the networks.

Figure 4 shows the synchronizability of multilayer star networks varying with the intralayer coupling strength $a$. From Figure 4(a), it is obvious that $\lambda_{2}$ increases linearly from zero and then remains invariant; this means that the synchronizability is strengthened at first and then remains 


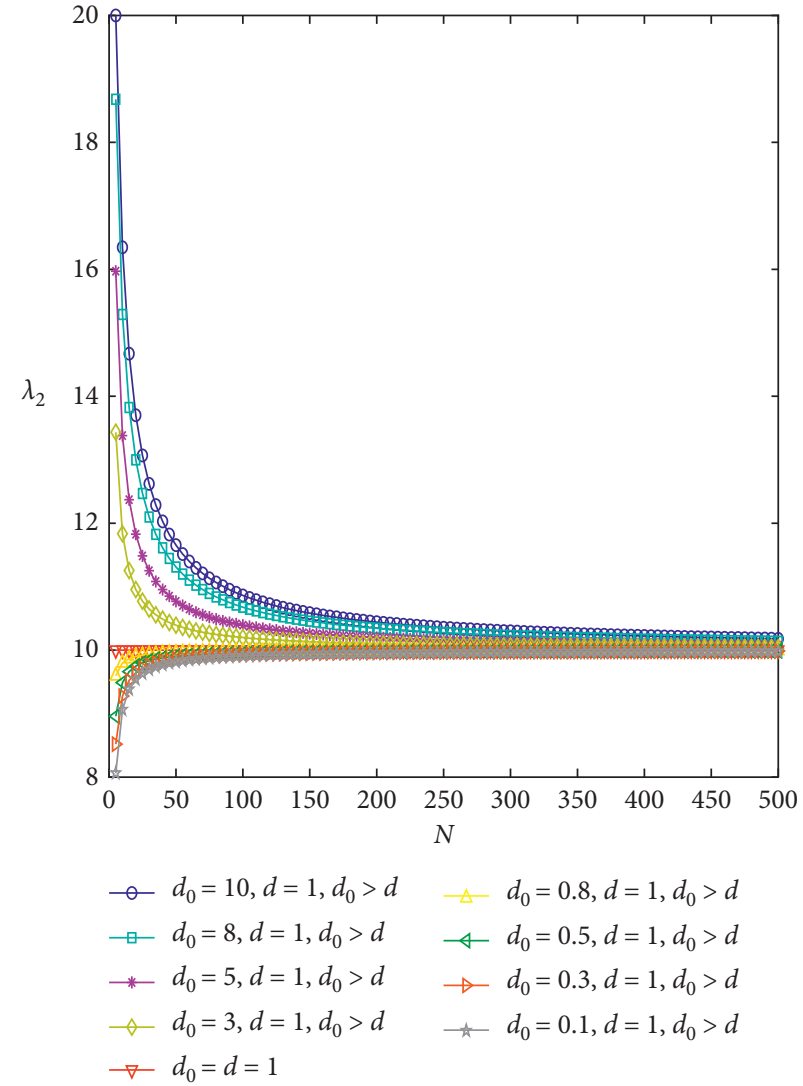

(a)

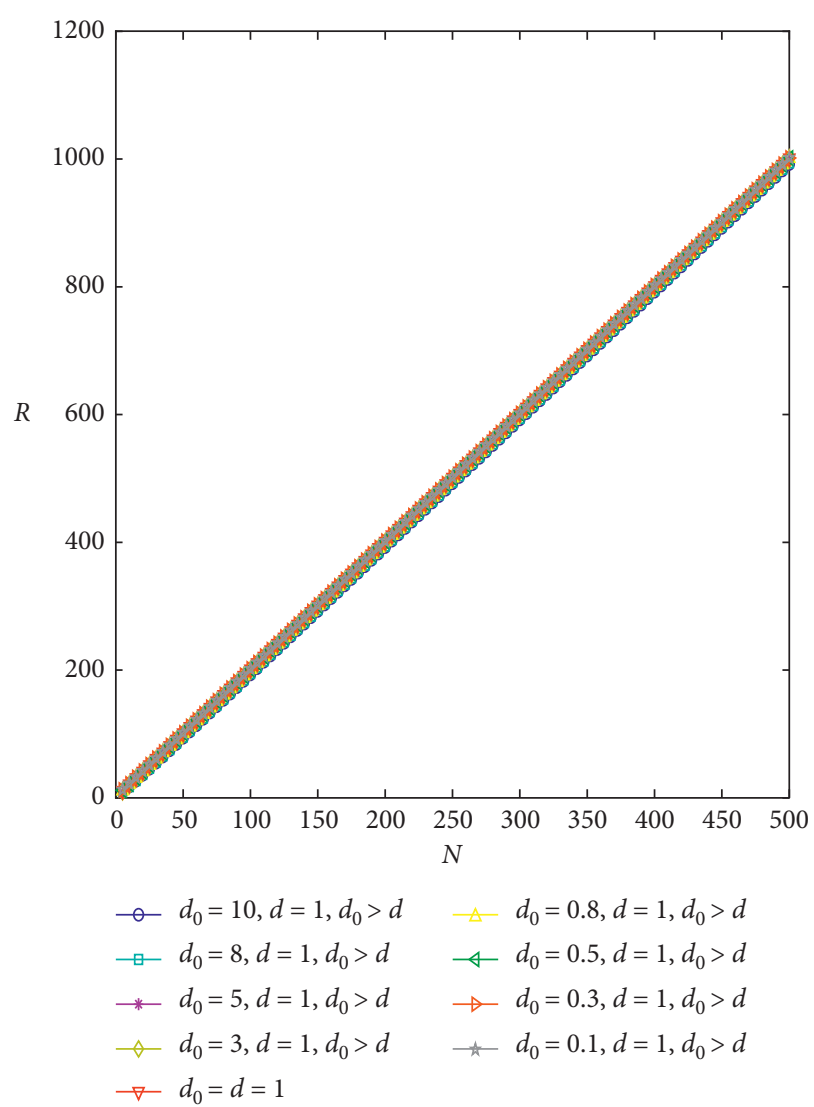

(b)

Figure 3: The synchronizability of multilayer star networks $v s$. varying the number of nodes $N(a=20, M=10, d=1$, and a $>M d)$. (a) $\lambda_{2}$ with respect to varying $N$ for different $d_{0}$. (b) $R$ with respect to varying $N$ for different $d_{0}$.

invariant with increasing $a$. When $a<M d$, different values of $d_{0}$ have no effect on the synchronizability of the networks; when $a>M d$, the smaller $d_{0}$, the worse the synchronizability, with the unbounded synchronous region. From Figure 4(b), one can observe that, with increasing a, $R$ first decreases (when $a<M d$ ) and then increases sharply (when $a>M d$ ), and the synchronizability is strengthened at first $(a<M d)$ and then weakened with increasing intralayer coupling strength $(a>M d)$. When $a<M d$, the smaller $d_{0}$, the stronger the synchronizability, and when $a>M d$, different values of $d_{0}$ have no effect on the synchronizability, with the bounded synchronous region. This is because when the intralayer coupling strength far exceeds the interlayer coupling strength, the structure of the multiplex network is similar to the community structure of multiple clusters; therefore, the synchronizability is weakened.

Figures 5(a) and 5(b) show the synchronizability is strengthened at first (when $a>M d$ ) and then remains invariant (when $a<M d$ ) with increasing $d$. That is to say, whether the synchronous region is unbounded or bounded, when $d<a / M$, the synchronizability of the networks is strengthened, and when $d>a / M$, the synchronizability of the networks remains basically invariant; therefore, there exists an optimal value of $d=a / M$ for interlayer coupling strength to maximizing synchronizability of multilayer star networks. When $d$ is too large, it not conducive to synchronization.

As is shown in Figure 6, when $a<M d$, panel (a) plots that the synchronizability is not affected by the interlayer coupling strength $d_{0}$, with the unbounded synchronous region; panel (b) plots that the synchronizability is weakened with $d_{0}$, with the bounded synchronous region. From Figure 7 (a), when $a>M d$, the synchronizability is strengthened slightly and basically maintained at $\lambda_{2}=M d=10$, with the unbounded synchronous region; from Figure 7(b), the synchronizability is also strengthened slightly, with the bounded synchronous region. Figure 8(a) shows that increasing the number of layers $M$ increases $\lambda_{2}$ (increases gradually at first and then approaches $M d$ ), and therefore, the synchronizability is strengthened at first and then basically maintained invariant, with the unbounded synchronous region. Figure 8(b) shows that the synchronizability is strengthened at first and then weakened slightly with increasing the number of layers, with the bounded synchronous region.

5.2. The Synchronizability of Multilayer Star-Ring Networks. For different interlayer coupling strength (i.e., $d_{0}>d, d_{0}<d$, and $d_{0}=d$ ), the synchronizability of the multilayer star-ring networks varies with the structural parameters, as shown in Figures 9-15. 


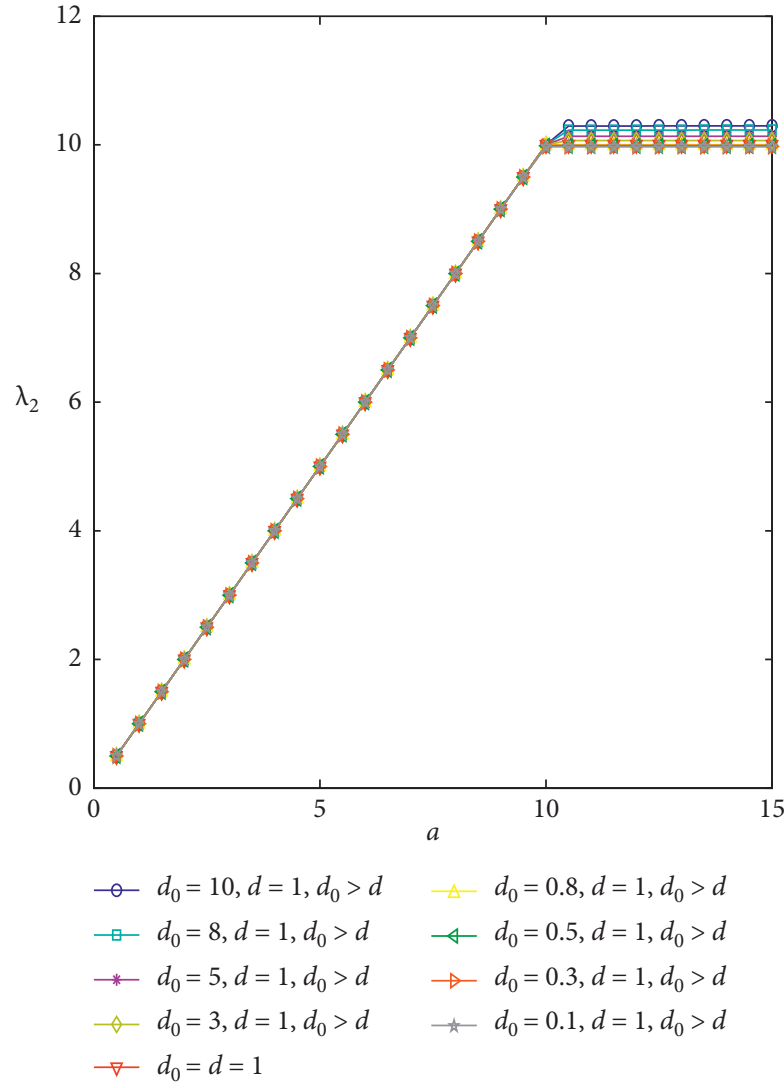

(a)

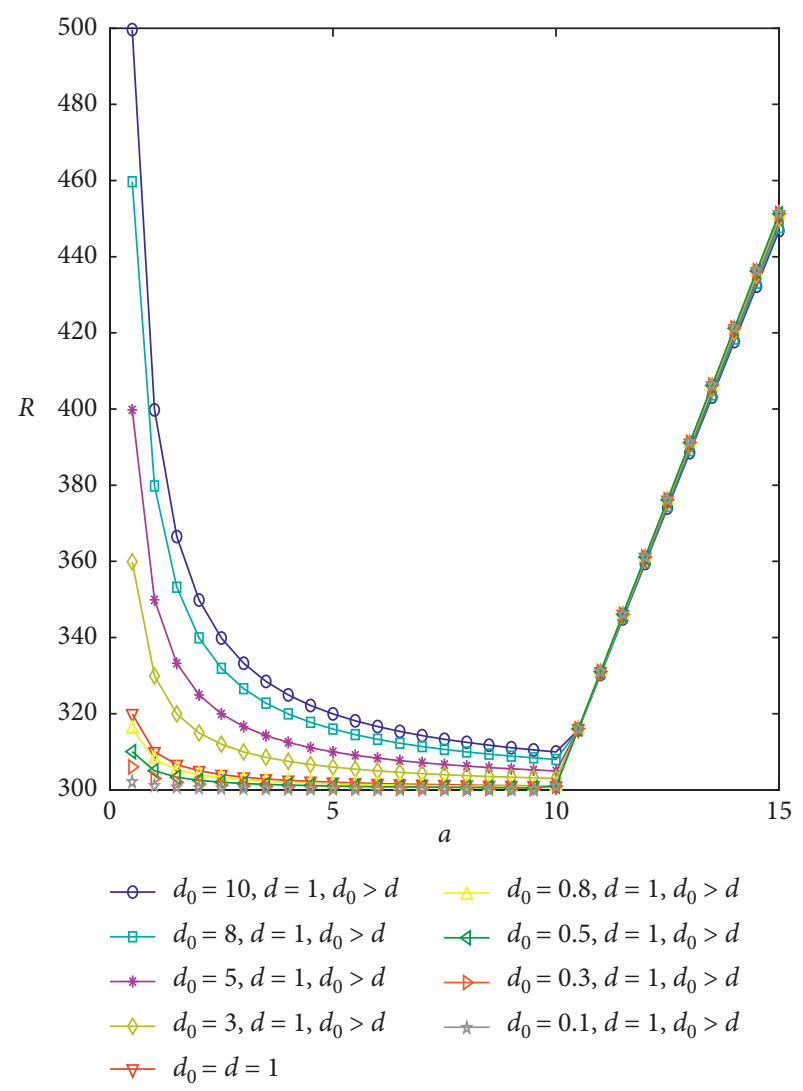

(b)

FIgURE 4: The synchronizability of multilayer star networks $v$ s. varying the intralayer coupling strength $a(N=300, M=10$, and $d=1)$. (a) $\lambda_{2}$ with respect to varying $a$ for different $d_{0}$. (b) $R$ with respect to varying $a$ for different $d_{0}$.

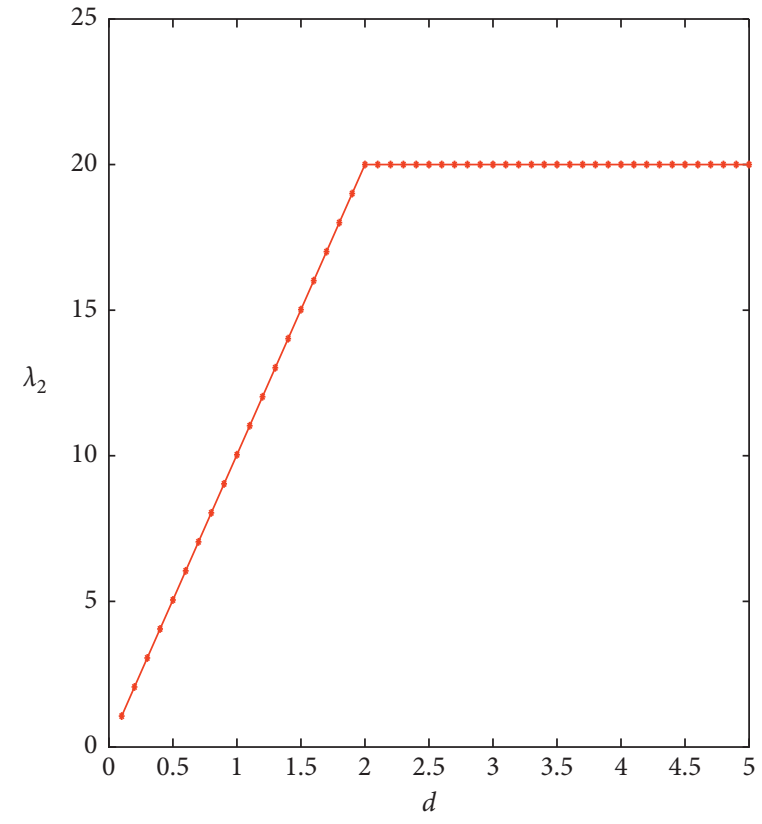

(a)

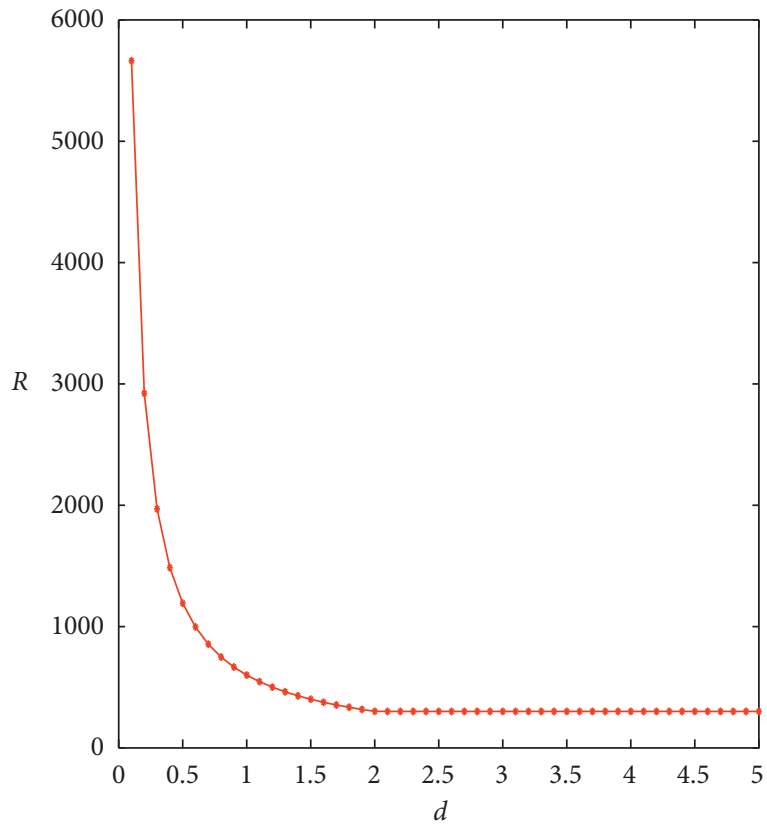

(b)

FIGURE 5: The synchronizability of multilayer star networks $v$ s. varying the interlayer coupling strength $d(N=300, \mathrm{M}=10, a=20$, and $\mathrm{d}_{0}=2$ ). (a) $\lambda_{2}$ with respect to varying $d$. (b) $R$ with respect to varying $d$. 


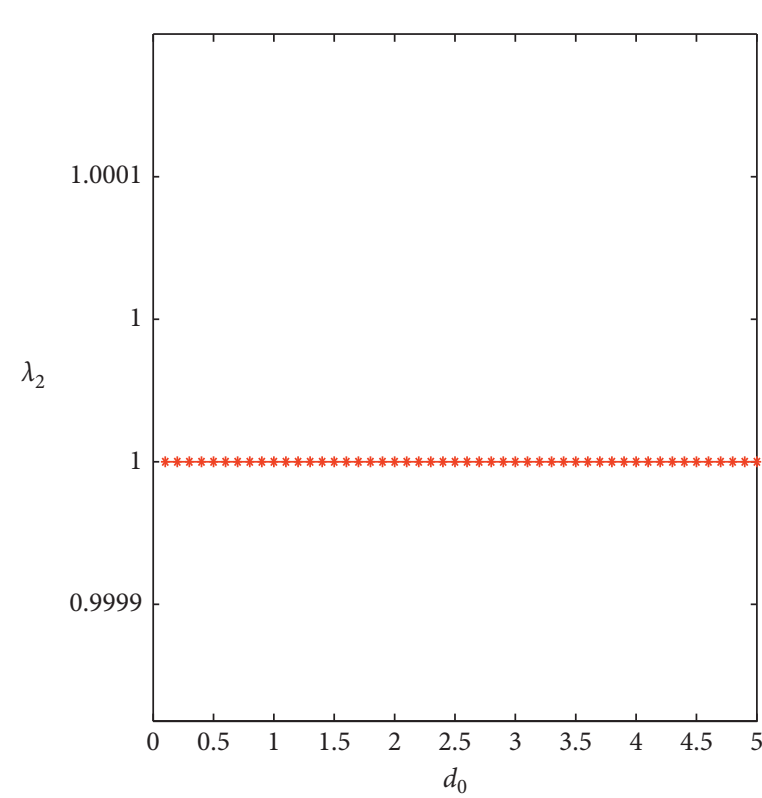

(a)

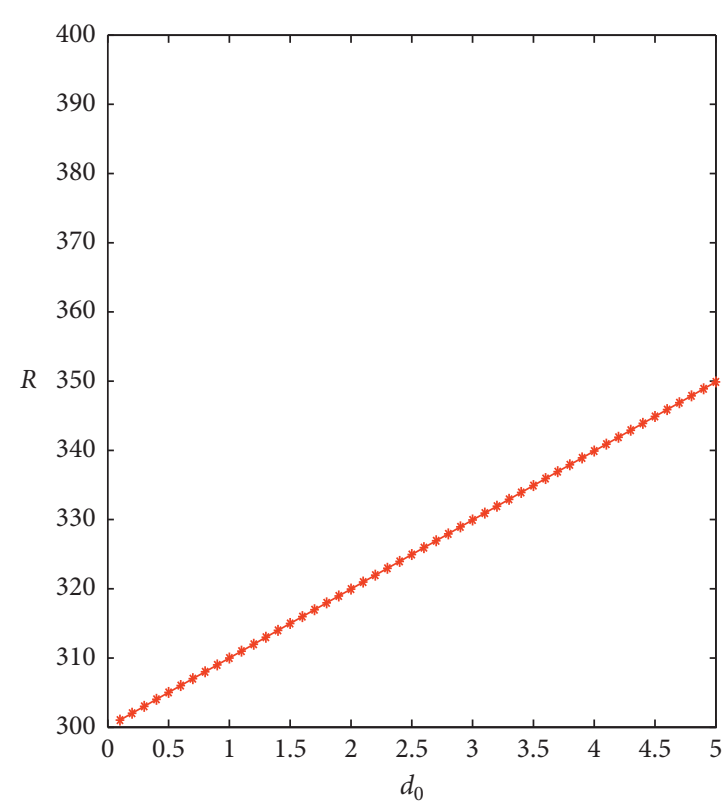

(b)

FIgURE 6: The synchronizability of multilayer star networks $v s$. varying the interlayer coupling strength $d_{0}(N=300, M=10, a=1, d=1$, and $a<M d$ ). (a) $\lambda_{2}$ with respect to varying $d_{0}$. (b) $R$ with respect to varying $d_{0}$.

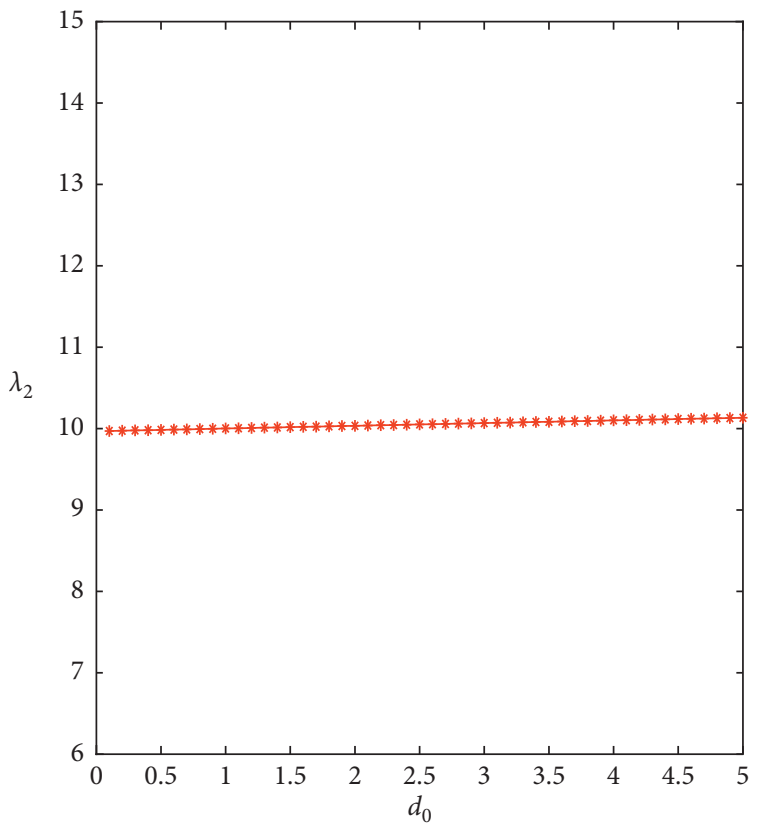

(a)

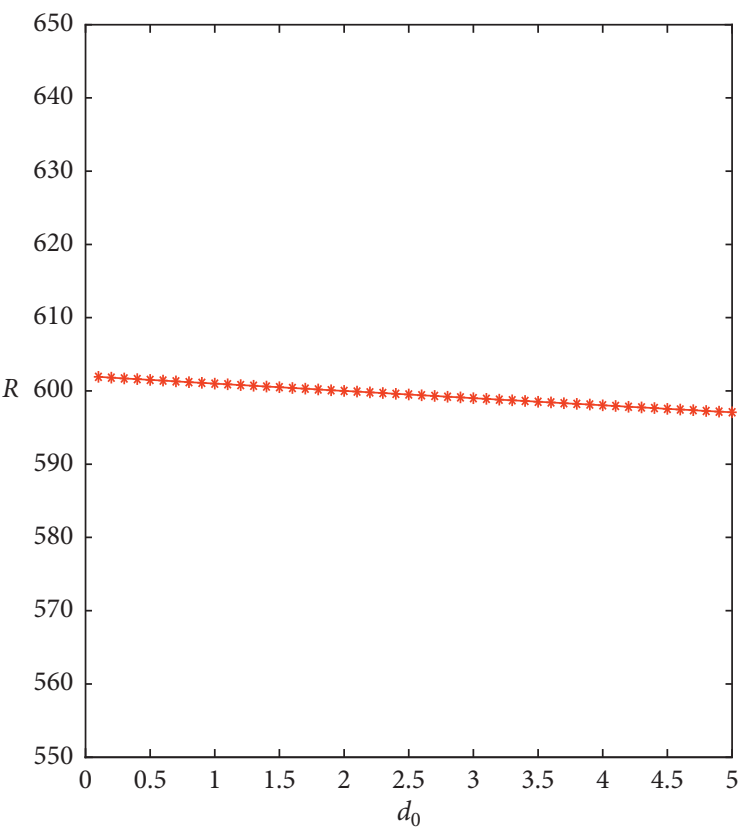

(b)

FIGURE 7: The synchronizability of multilayer star networks $v s$. varying the interlayer coupling strength $d_{0}(N=300, M=10, a=20, d=1$, and $a>M d$ ). (a) $\lambda_{2}$ with respect to varying $d_{0}$. (b) $R$ with respect to varying $d_{0}$.

From Figure 9, when $a<M d$, the synchronizability of multilayer star-ring networks is weakened at first and then remains invariant with increasing $N$, with the unbounded synchronous region. When the synchronous region is bounded, the synchronizability is weakened with increasing the number of nodes in each layer $N$.

Figure 10 displays that the synchronizability remains invariant with increasing $N$ for $d_{0}=d$; the synchronizability 


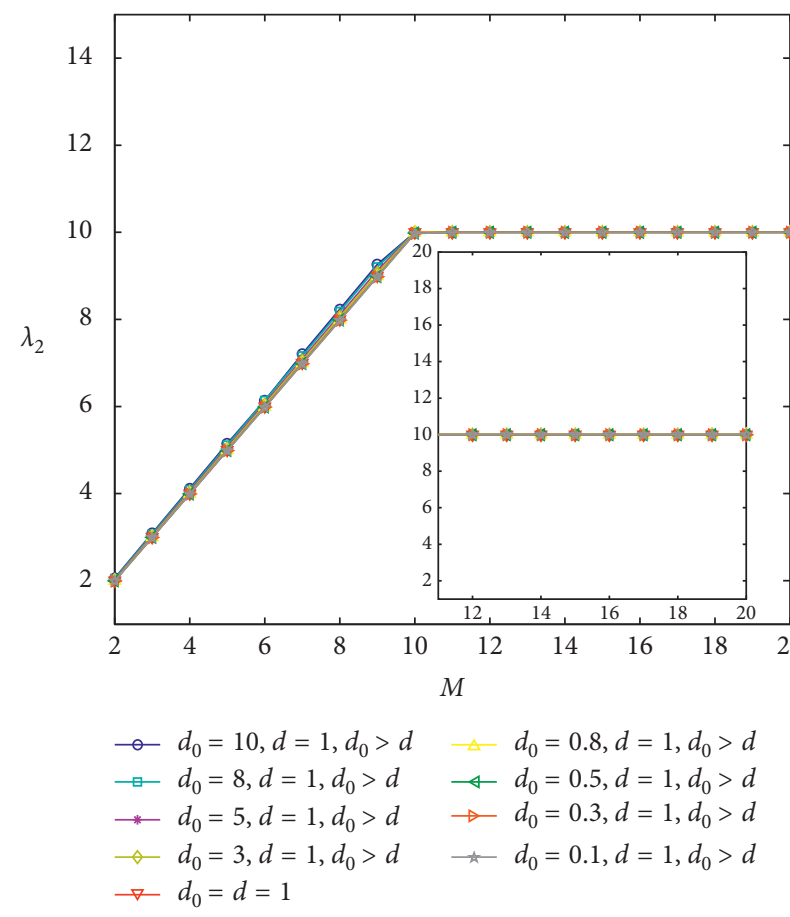

(a)

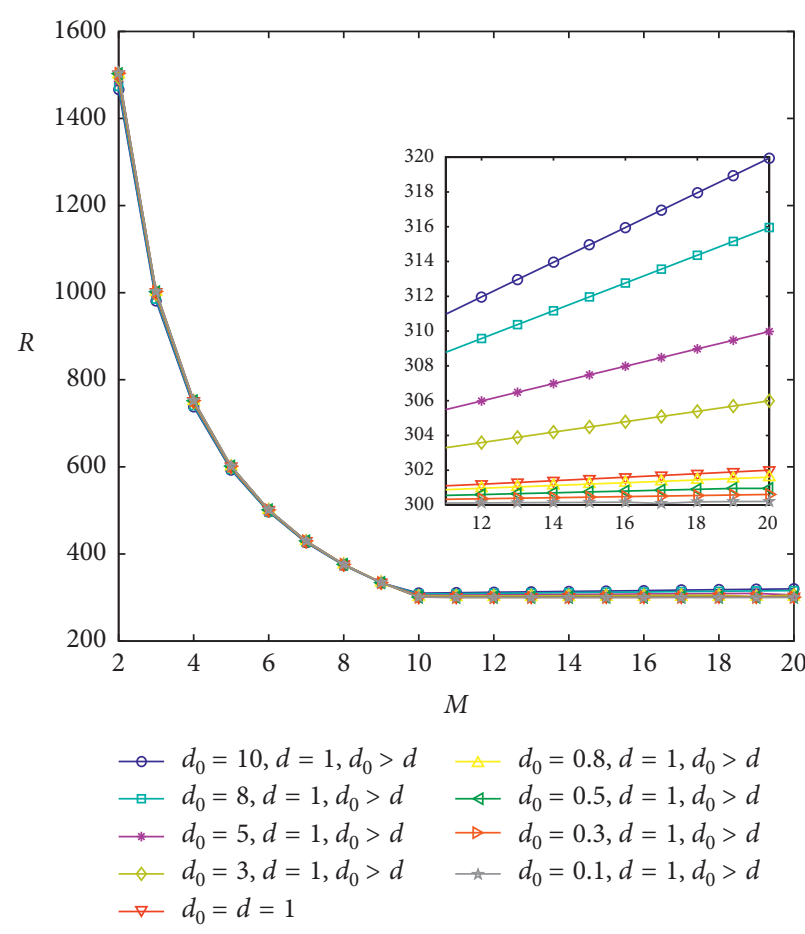

(b)

FIGURE 8: The synchronizability of multilayer star networks $v s$. varying the number of the layers $M(N=100, a=10$, and $d=1)$. (a) $\lambda_{2}$ with respect to varying $M$ for different $d_{0}$. (b) $R$ with respect to varying $M$ for different $d_{0}$.

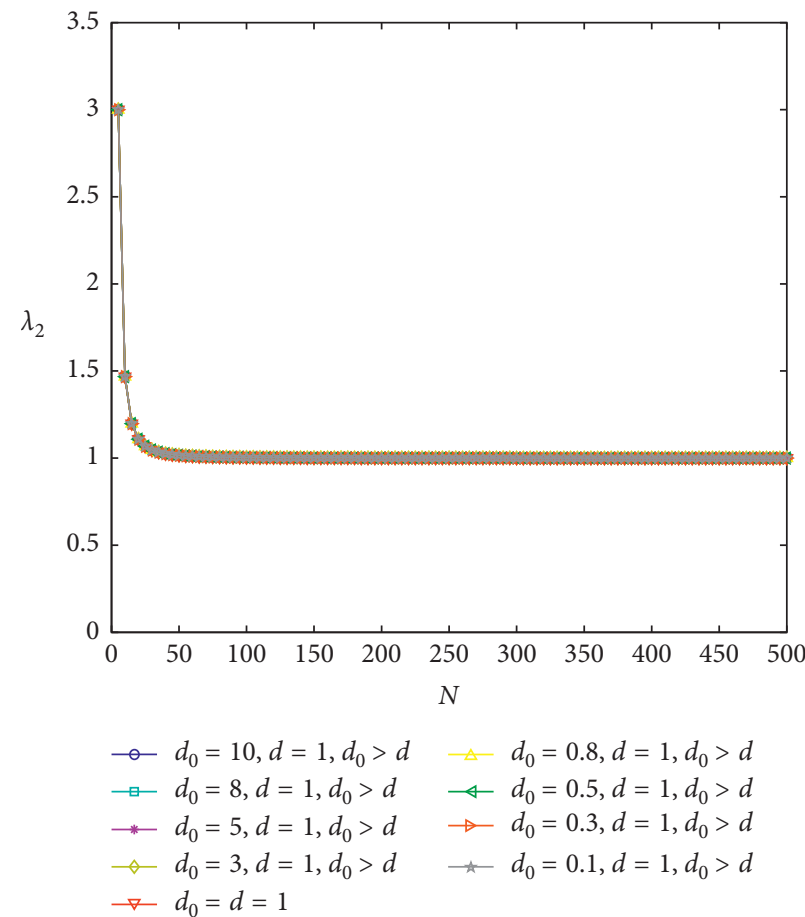

(a)
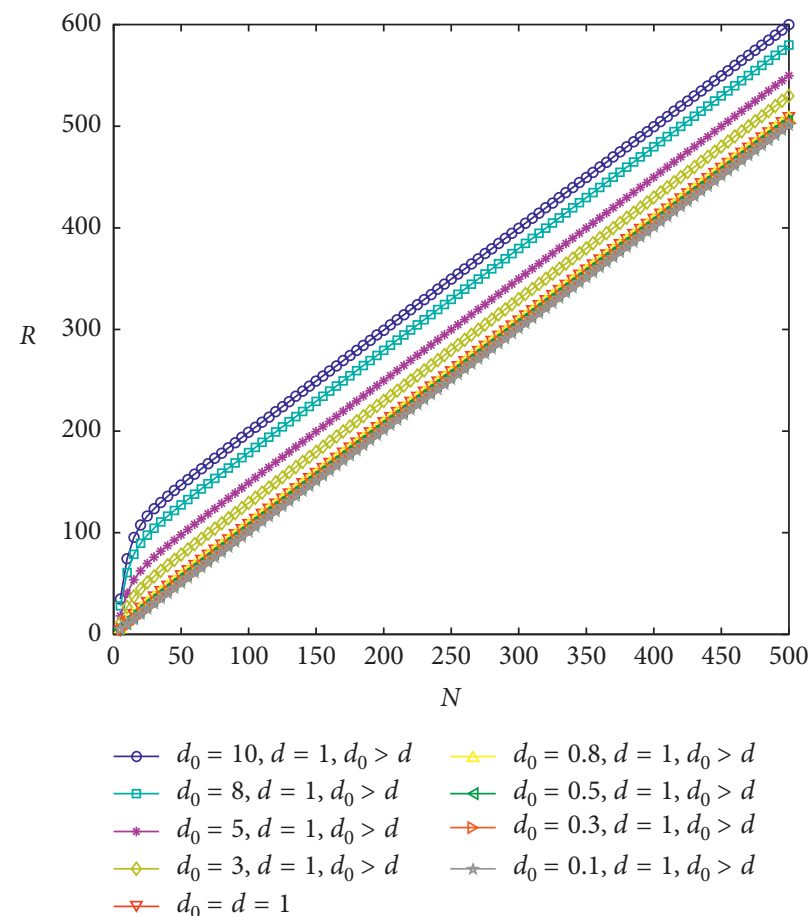

(b)

FIgURE 9: The synchronizability of multilayer star-ring networks $v$. varying the number of nodes $N(a=1, M=10, d=1$, and a $<M d)$. (a) $\lambda_{2}$ with respect to varying $N$ for different $d_{0}$. (b) $R$ with respect to varying $N$ for different $d_{0}$. 


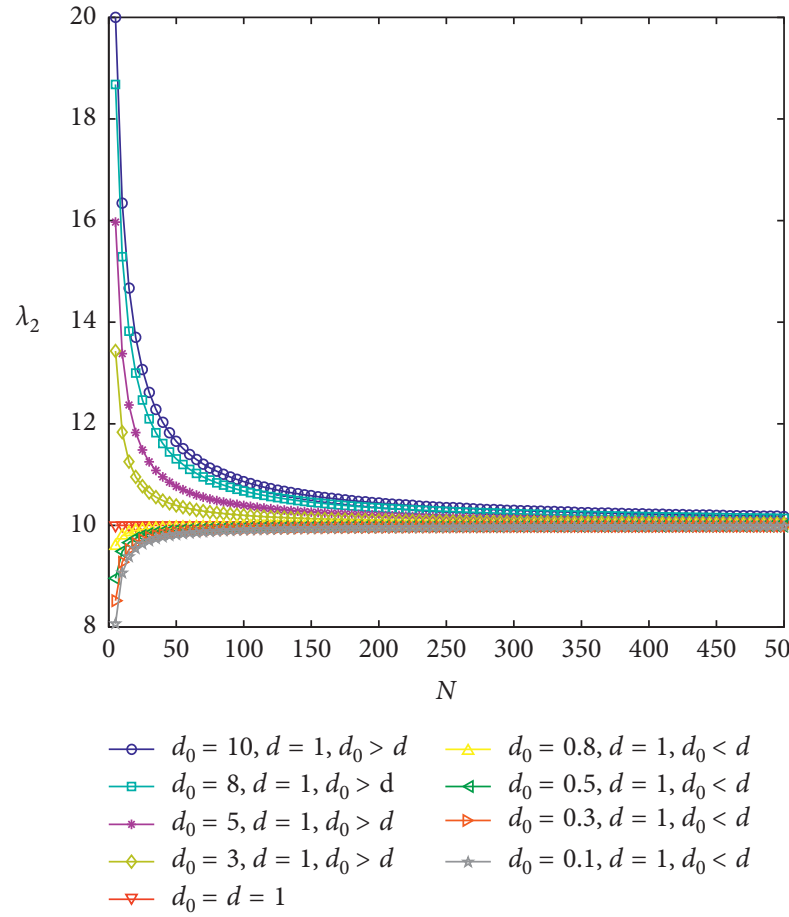

(a)

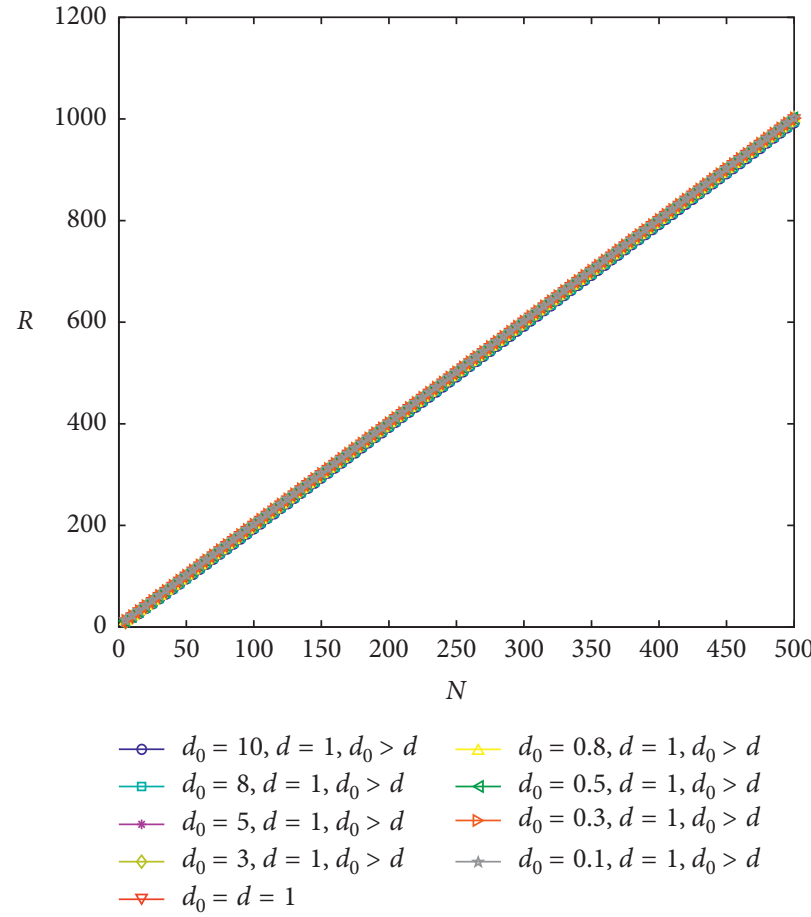

(b)

FiguRE 10: The synchronizability of multilayer star-ring networks $v s$. varying the number of nodes $N(a=20, M=10, d=1$, and $a>M d)$. (a) $\lambda_{2}$ with respect to varying $N$ for different $d_{0}$. (b) $R$ with respect to varying $N$ for different $d_{0}$.

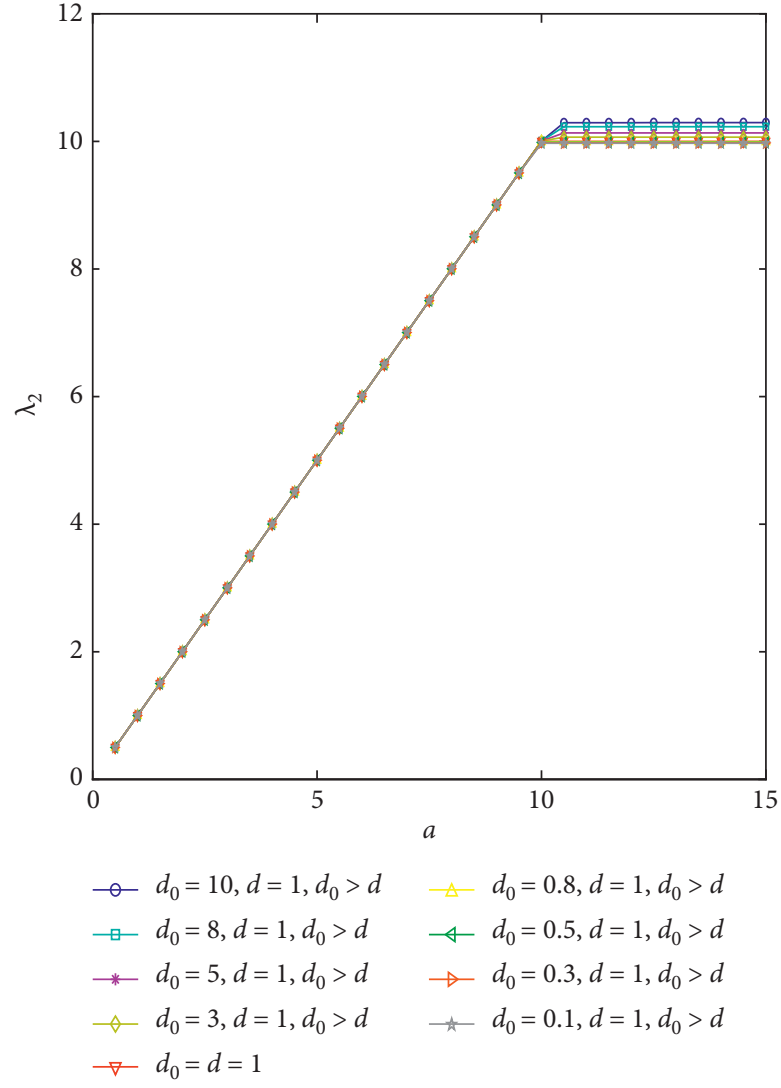

(a)

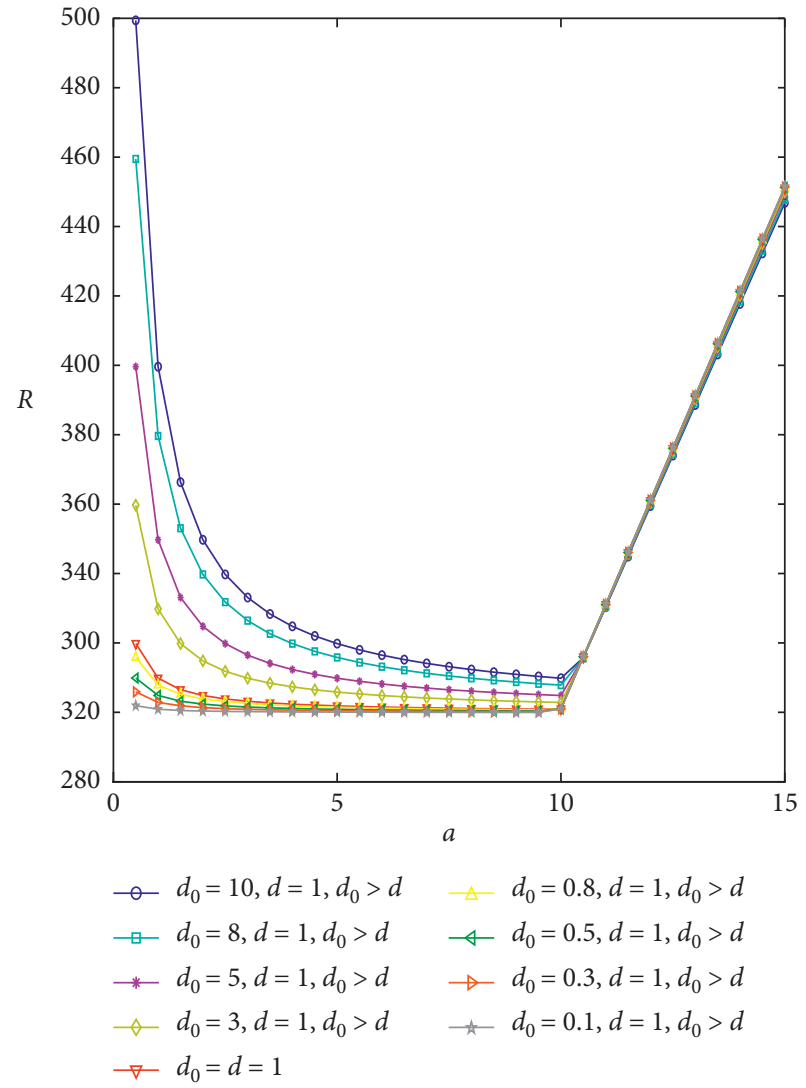

(b)

FiguRE 11: The synchronizability of multilayer star-ring networks $v$ s. varying the intralayer coupling strength $a(N=300, M=10$, and $d=1)$. (a) $\lambda_{2}$ with respect to varying $a$ for different $d_{0}$. (b) $R$ with respect to varying $a$ for different $d_{0}$. 


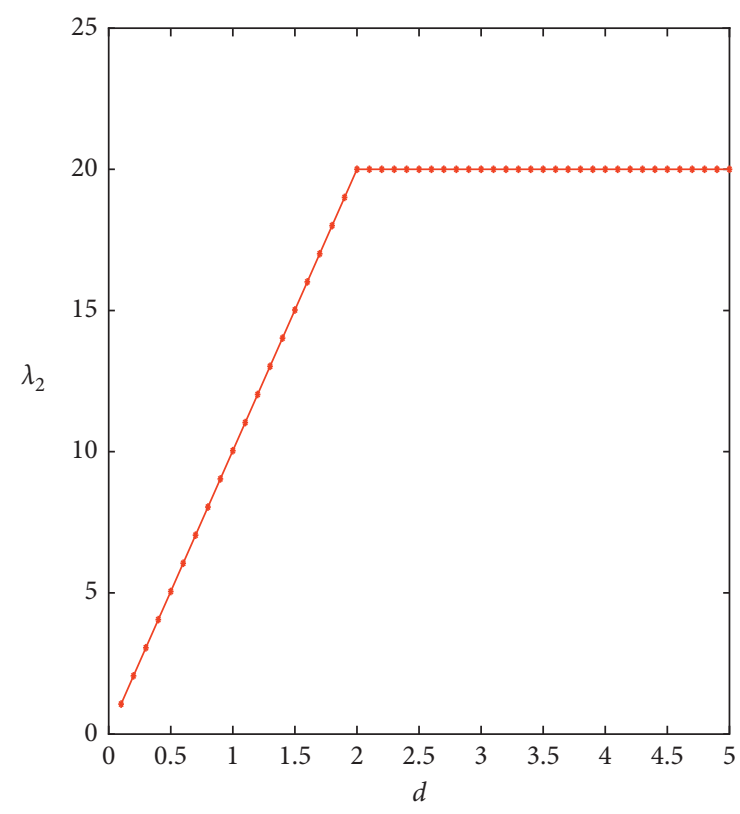

(a)

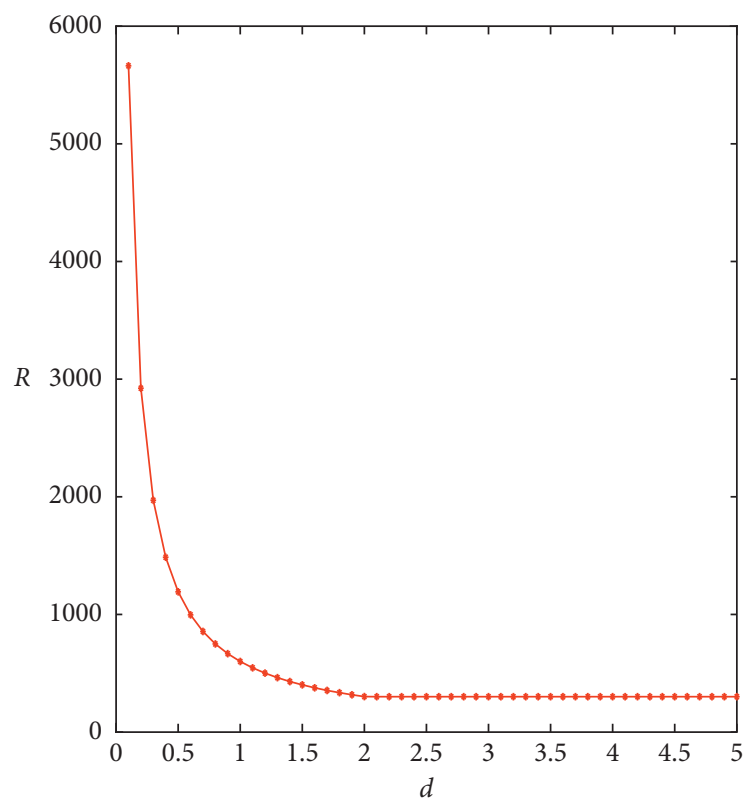

(b)

FIGURE 12: The synchronizability of multilayer star-ring networks $v$ s. varying the interlayer coupling strength $d(N=300, M=10, a=20$, and $d_{0}=2$ ). (a) $\lambda_{2}$ with respect to varying $d$. (b) $R$ with respect to varying $d$.

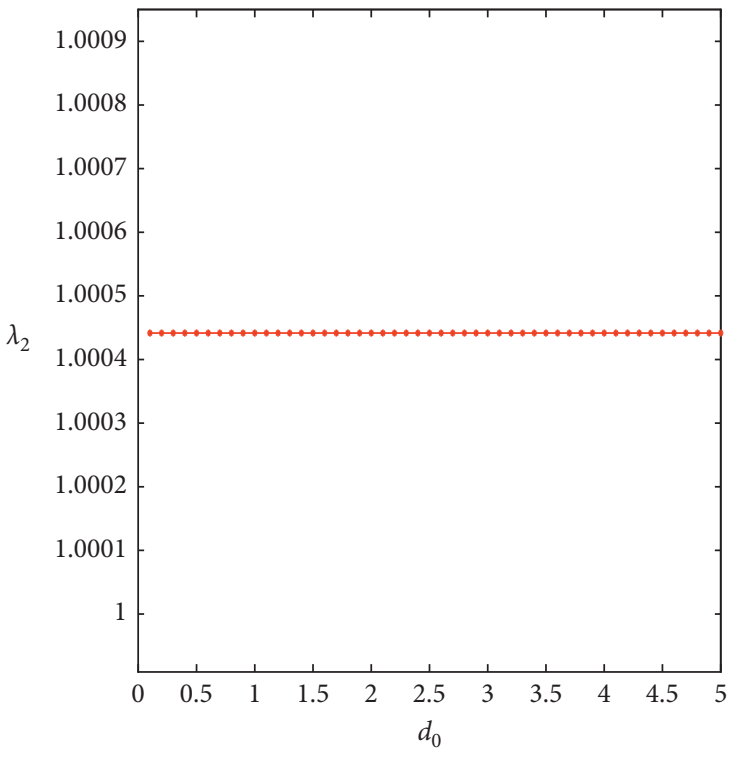

(a)

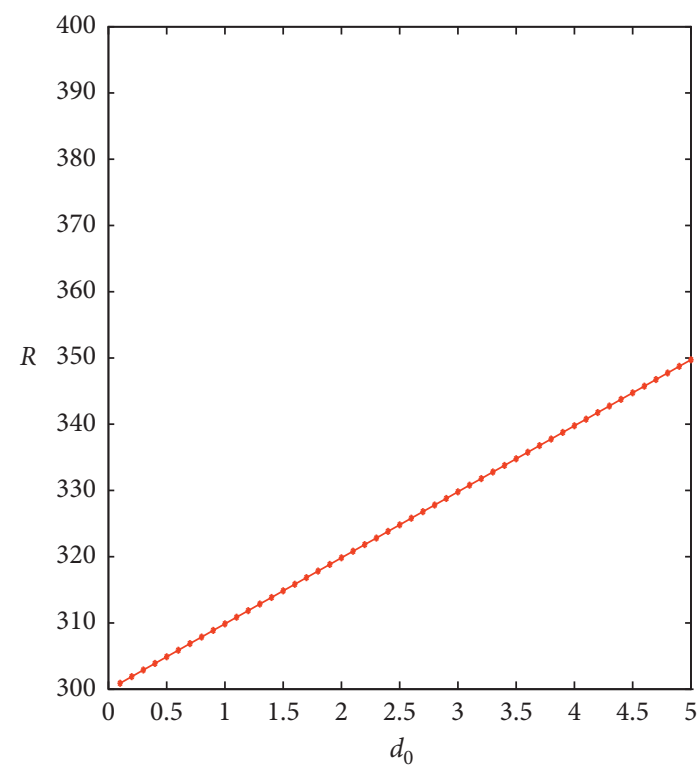

(b)

FIGURE 13: The synchronizability of multilayer star-ring networks $v$ s. varying the interlayer coupling strength $d_{0}(N=300, M=10, a=1, d=$ 1 , and $a<M d$ ). (a) $\lambda_{2}$ with respect to varying $d_{0}$. (b) $R$ with respect to varying $d_{0}$.

is strengthened at first and then almost remains invariant with increasing $N$ for $d_{0}<d$; and the synchronizability is weakened at first and then almost remains invariant with increasing $N$ for $d_{0}>d$, with the unbounded synchronous region. The influences of the number of nodes on synchronizability for the multilayer star-ring networks are similar to those for the multilayer star networks. When the synchronous region is bounded, the synchronizability is also weakened with increasing the number of nodes in each layer $N$.

As can be obtained from Figures 11-15, the influences of the structural parameters (i.e., the number of layers, the interlayer coupling strength, and the intralayer coupling strength) on synchronizability for the multilayer star-ring networks are basically similar to those for the multilayer star networks; a few different points between the two will be introduced in Section 5.3. 


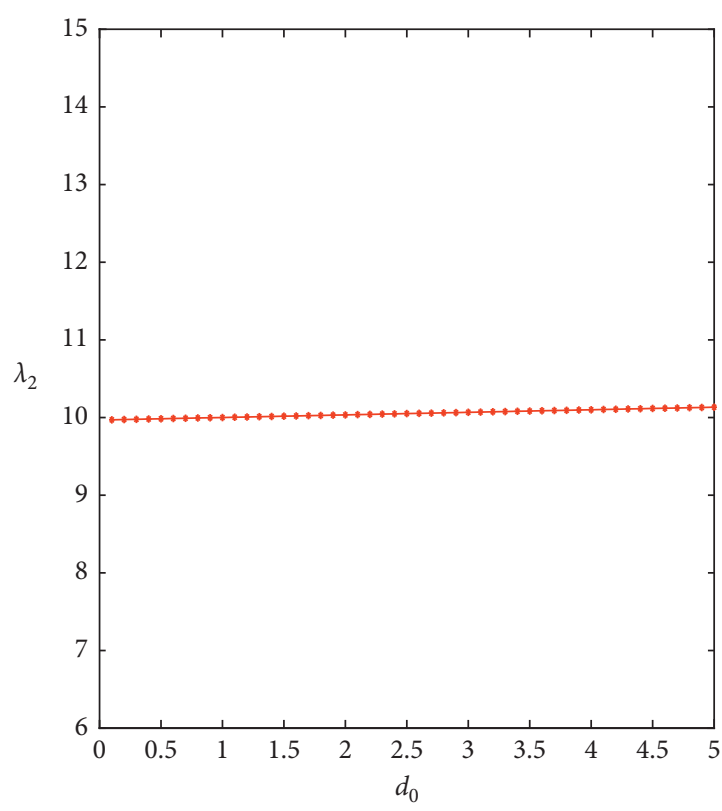

(a)

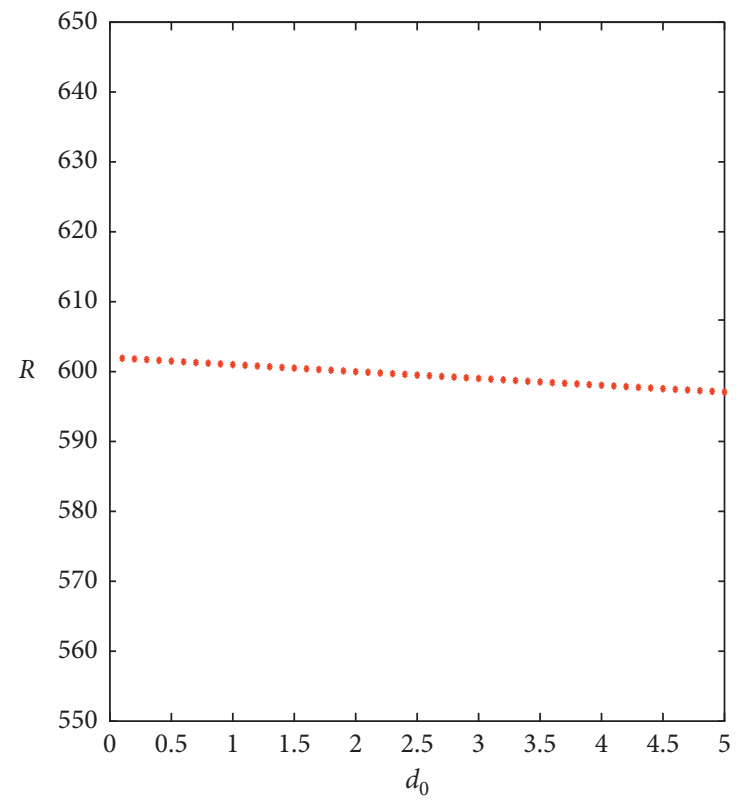

(b)

FIGURE 14: The synchronizability of multilayer star-ring networks $v$ s. varying the interlayer coupling strength $d_{0}(N=300, M=10, a=20, d=$ 1 , and $a>M d$ ) (a) $\lambda_{2}$ with respect to varying $d_{0}$. (b) $R$ with respect to varying $d_{0}$.

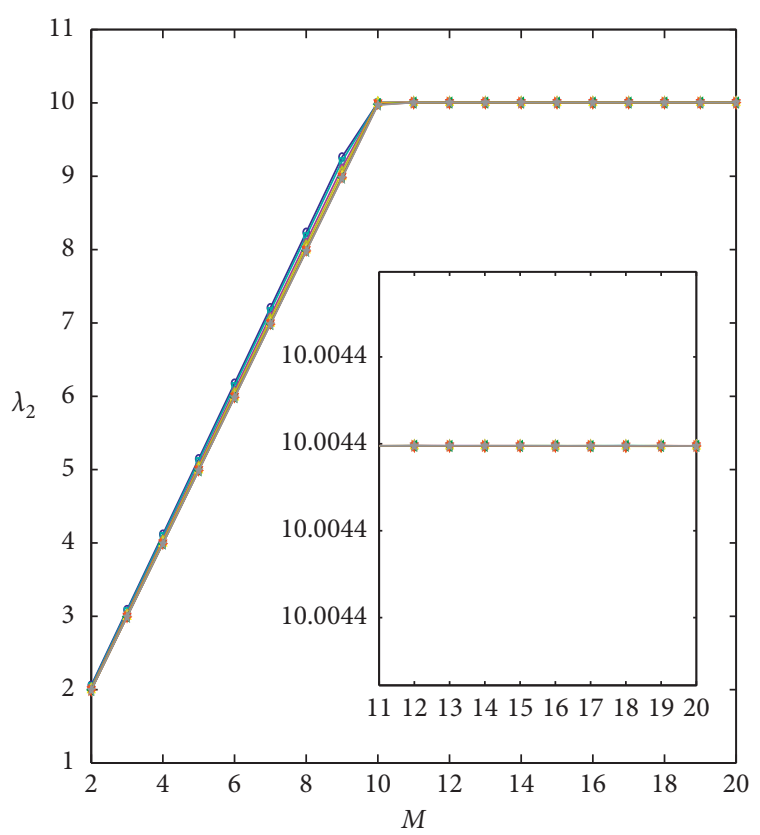

$$
\begin{aligned}
& \rightarrow d_{0}=10, d=1, d_{0}>d \quad \rightarrow d_{0}=0.8, d=1, d_{0}<d \\
& \rightarrow d_{0}=8, d=1, d_{0}>d \quad \rightarrow d_{0}=0.5, d=1, d_{0}<d \\
& \rightarrow d_{0}=5, d=1, d_{0}>d \quad \rightarrow d_{0}=0.3, d=1, d_{0}<d \\
& \rightarrow d_{0}=3, d=1, d_{0}>d \quad \rightarrow d_{0}=0.1, d=1, d_{0}<d \\
& \rightarrow d_{0}=d=1
\end{aligned}
$$

(a)

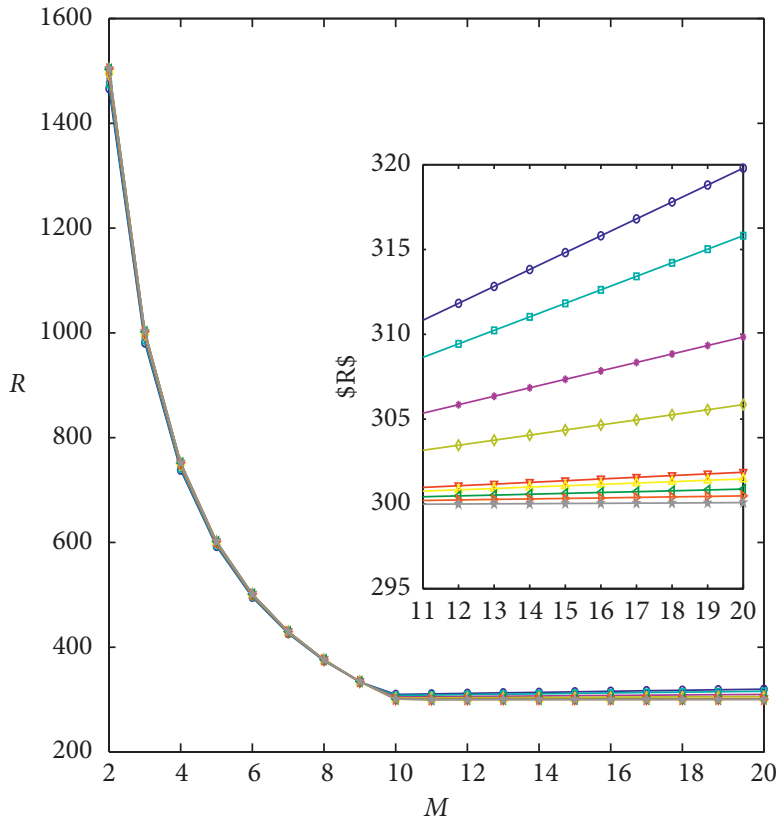

$$
\begin{aligned}
& \rightarrow d_{0}=10, d=1, d_{0}<d \quad \rightarrow d_{0}=0.8, d=1, d_{0}<d \\
& \rightarrow d_{0}=8, d=1, d_{0}<d \quad \rightarrow d_{0}=0.5, d=1, d_{0}<d \\
& \rightarrow d_{0}=5, d=1, d_{0}<d \quad \rightarrow d_{0}=0.3, d=1, d_{0}<d \\
& \rightarrow d_{0}=3, d=1, d_{0}<d \quad \rightarrow d_{0}=0.1, d=1, d_{0}<d \\
& \rightarrow d_{0}=d=1
\end{aligned}
$$

(b)

FIGURE 15: The synchronizability of multilayer star-ring networks $v s$. varying the number of the layers $M(N=100, a=10$, and $d=1)$. (a) $\lambda_{2}$ with respect to varying $M$ for different $d_{0}$. (b) $R$ with respect to varying $M$ for different $d_{0}$. 


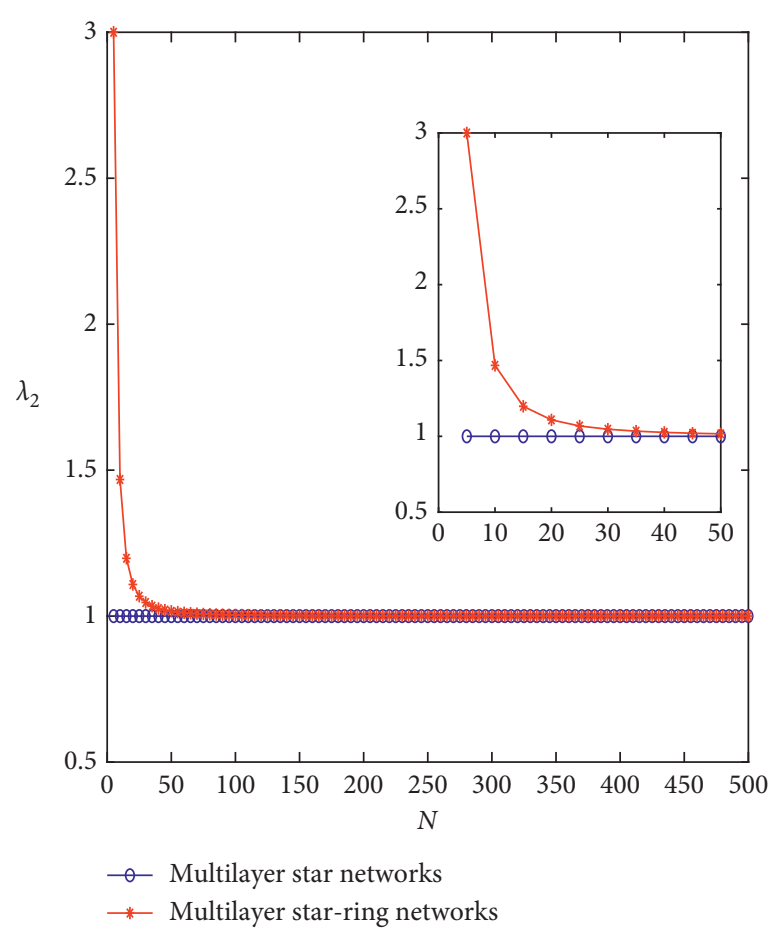

(a)

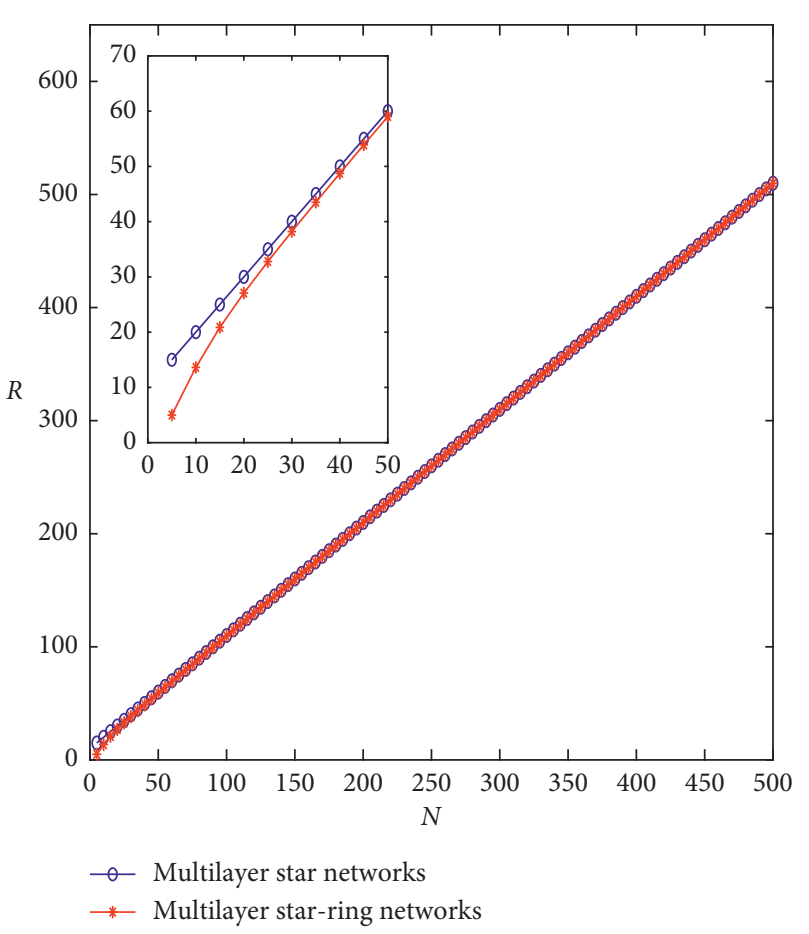

(b)

FIgURE 16: The synchronizability of multilayer star and star-ring networks, where $a=1, d=1, M=10$, and $a<M d$. (a) $\lambda_{2}$ with respect to varying $N$ for multilayer star and star-ring networks. (b) $R$ with respect to varying $N$ for multilayer star and star-ring networks.

5.3. Comparison of Two Types of Network Synchronizability. In this section, we will discuss the difference of synchronizability between multilayer star and star-ring networks under the same structural parameters.

Figure 16 shows $\lambda_{2}$ and eigenratio $R$ numerically calculated from supra-Laplacian matrices of multilayer star and star-ring networks with varying scale size $N$ in the case of $a<$ $M d$. When $N$ is small (approximately $N<50$ ), as can be observed from Figure 16(a), $\lambda_{2}$ for multilayer star-ring networks is larger than that for multilayer star networks; Figure 16(b) shows $R$ for multilayer star networks is larger than that for multilayer star-ring networks. This implies that, when $N$ is small, the multilayer star-ring network has better synchronizability as that of the multilayer star network. When $N$ is sufficiently large, the multilayer star-ring network has the same synchronizability as that of the multilayer star network, and the synchronizability of the two types of networks is nearly invariant with increasing $N$, implying that a larger size has little effect on the synchronizability of the two networks, for the unbounded synchronous region; while leads to linearly weakened synchronizability for two types of networks, for the bounded synchronous region. It is obvious that the simulation results are consistent with the theoretical results, for the case of $a<M d$; when $N$ is small enough, $\lambda_{2}=$ $a+4 a \sin ^{2}(\pi / N-1)$ for multilayer star-ring networks is larger than $\lambda_{2}=a$ for multilayer star networks; when $N$ is sufficiently large, $\lambda_{2}=a+4 a \sin ^{2}(\pi / N-1) \longrightarrow a$ of multilayer star-ring networks is almost equal to that of multilayer star networks.
Figure 17 shows $\lambda_{2}$ and eigenratio $R$ numerically calculated from supra-Laplacian matrices of multilayer star and star-ring networks with varying scale size $N$ in the case of $a>M d$. When $a>M d$, the multilayer star-ring network has the same synchronizability as that of the multilayer star network under taking the same structural parameter values. The synchronizability of the two networks is determined by the number of the layers $M$ and the interlayer coupling strength $d$ between leaf nodes and invariant with increasing $N$, for the unbounded synchronous region; the synchronizability of the two networks is weakened with increasing $N$, for the bounded synchronous region. The simulation results are consistent with the theoretical results.

\section{Discussion and Conclusion}

In this paper, the eigenvalue spectrum of the supraLaplacian matrices of multilayer star and star-ring networks is strictly derived theoretically, and the synchronizability of two kinds of multilayer networks varying with the structural parameters is analyzed. In the case of unbounded synchronous region, the synchronizability of two types of networks is related to the intralayer coupling strength and interlayer coupling strength (the network scale is fixed) under different interlayer coupling strength. When the intralayer coupling strength $a$ is relatively weak, the synchronizability of the networks only depends on the intralayer coupling strength $a$. When the interlayer coupling strength $d$ between the leaf nodes is weak, the 


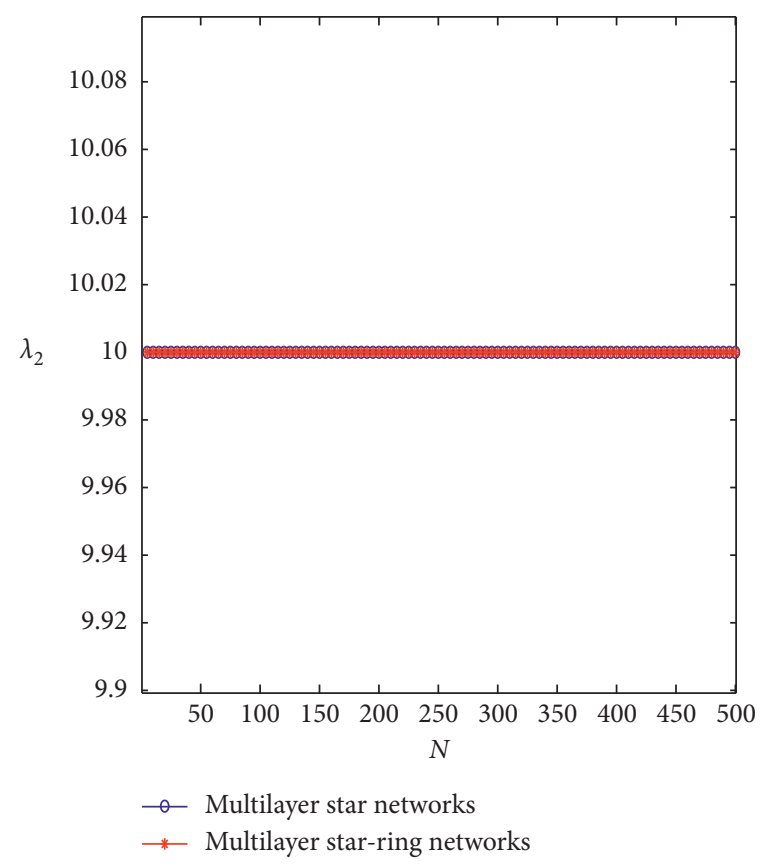

(a)

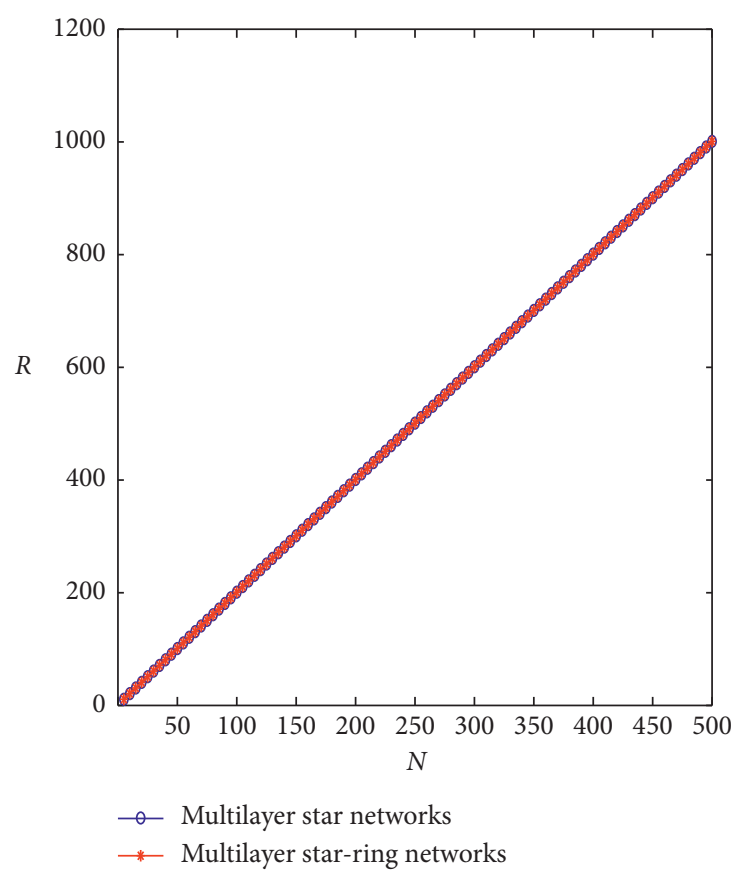

(b)

FIgURE 17: The synchronizability of multilayer star and star-ring networks, where a $=20, d=1, M=10$, and $a>M d$. (a) $\lambda_{2}$ with respect to varying $N$ for multilayer star and star-ring networks. (b) $R$ with respect to varying $N$ for multilayer star and star-ring networks.

synchronizability only depends on the interlayer coupling strength $d$, that is, the synchronizability is determined by the weaker of the two. In the case of the bounded synchronous region, when the intralayer coupling strength is weak, the increase of the intralayer coupling strength will enhance the synchronizability of the networks, while the increase of the interlayer coupling strength between the leaf nodes will weaken the synchronizability. When the interlayer coupling strength between the leaf nodes $d$ is weak, the increase of the interlayer coupling strength between the leaf nodes will enhance the synchronizability of the networks, while the increases of the intralayer coupling strength will weaken the synchronizability. The same change of the intralayer coupling strength and the interlayer coupling strength between the leaf nodes has an opposite effect on the synchronizability of the two types of networks. Therefore, when the scale of the networks is fixed ( $M$ and $N$ are unchanged), the interlayer coupling strength $d$ increases, we can increase the intralayer coupling strength $a$ to maintain the synchronizability. The multilayer star-ring networks have better synchronizability than that of multilayer star networks, for sufficiently small $N$.

So far, there are many unresolved issues in the research studies on the multilayer network. For example, for the multilayer star network and star-ring network proposed in this paper, how to adjust or control the relationship between parameters $d, d_{0}$, and $a$ to maintain or improve the synchronizability of the networks? How to optimize the structural parameters of the networks to achieve the best synchronizability? All these need further study. In a word, many theoretical and practical problems have to be challenged.

\section{Data Availability}

The data used to support the findings of this study are included within the supplementary information file.

\section{Conflicts of Interest}

The authors declare that they have no conflicts of interest.

\section{Acknowledgments}

This project was supported by the Natural Science Foundation of Guangxi (no. 2018GXNSFAA138095) and the National Natural Science Foundation of China (no. 61563013).

\section{Supplementary Materials}

The procedures are MATLAB language code, and the data are obtained by running the code provided. (Supplementary Materials)

\section{References}

[1] L. Tang, J. Lu, X. Wu, and J. Lü, "Impact of node dynamics parameters on topology identification of complex dynamical networks," Nonlinear Dynamics, vol. 73, no. 1-2, pp. 1081-1097, 2013.

[2] X. F. Wang et al., "Complex networks: topology, dynamics and synchronization," International Journal of Bifurcation and Chaos, vol. 12, no. 5, pp. 885-916, 2002. 
[3] A. Barrat, M. Barthélemy, and A. Vespignani, "Weighted evolving networks: coupling topology and weight dynamics," Physical Review Letters, vol. 92, no. 22, Article ID 228701, 2004.

[4] W. Wang, B. Wang, B. Hu, G. Yan, and Q. Ou, "General dynamics of topology and traffic on weighted technological networks," Physical Review Letters, vol. 94, no. 18, Article ID 188702, 2005.

[5] H. Kohestani, M. Totonkuban, L. Di Paola, V. Todde, and A. Giuliani, "The basic principles of topology-dynamics relations in networks: an empirical approach," Physica A: Statistical Mechanics and its Applications, vol. 508, pp. 584-594, 2018.

[6] G. Mei, X. Wu, D. Ning, and J.-A. Lu, "Finite-time stabilization of complex dynamical networks via optimal control," Complexity, vol. 21, no. 1, pp. 417-425, 2016.

[7] F. Liu, Q. Song, and J. Cao, "Pinning synchronization of onesided lipschitz complex networks," Discrete Dynamics in Nature and Society, vol. 2014, Article ID 627060, 8 pages, 2014.

[8] F. Zhang, J. Jia, and L. Yang, "Adaptive synchronization in complex network with different order node dynamics," Discrete Dynamics in Nature and Society, vol. 2015, Article ID 919146, 7 pages, 2015.

[9] X. Liu and T. Chen, "Cluster synchronization in directed networks via intermittent pinning control," IEEE Transactions on Neural Networks, vol. 22, no. 7, pp. 1009-1020, 2011.

[10] Y. Yang, G. Xie, and J. Xie, "Mining important nodes in directed weighted complex networks," Discrete Dynamics in Nature and Society, vol. 2017, Article ID 9741824, 7 pages, 2017.

[11] S. Weber, M.-T. Hütt, and M. Porto, "Pattern formation and efficiency of reaction-diffusion processes on complex networks," EPL (Europhysics Letters), vol. 82, no. 2, Article ID 28003, 2008.

[12] G. Siudem and J. A. Hołyst, "Diffusion on hierarchical systems of weakly-coupled networks," Physica A: Statistical Mechanics and its Applications, vol. 513, pp. 675-686, 2019.

[13] M. Rounaghi and F. Zadeh, "Investigation of market efficiency and financial stability between S\&P 500 and London stock exchange: monthly and yearly forecasting of time series stock returns using ARMA model," Physica A: Statistical Mechanics and its Applications, vol. 456, pp. 10-21, 2016.

[14] A. Arenas, A. Díaz-Guilera, J. Kurths, Y. Moreno, and C. Zhou, "Synchronization in complex networks," Physics Reports, vol. 469, no. 3, pp. 93-153, 2008.

[15] M. Denker, M. Timme, M. Diesmann, F. Wolf, and T. Geisel, "Breaking synchrony by heterogeneity in complex networks," Physical Review Letters, vol. 92, no. 7, Article ID 074103, 2004.

[16] A. E. Motter, C. Zhou, and J. Kurths, "Network synchronization diffusion and the paradox of heterogeneity," Physical Review E, vol. 71, no. 1, Article ID 016116, 2005.

[17] M. Xue, E. Yeung, A. Rai et al., "Initial-condition estimation in network synchronization processes: algebraic and graphical characterizations of the estimator," Complex Systems, vol. 21, pp. 297-333, 2013.

[18] Z. Jia, L. Zeng, Y.-Y. Wang, and P. Wang, "Optimization algorithms for spectral coarse-graining of complex networks," Physica A: Statistical Mechanics and its Applications, vol. 514, pp. 925-935, 2019.

[19] Y.-S. Long, Z. Jia, and Y.-Y. Wang, "Coarse graining method based on generalized degree in complex network," Physica A: Statistical Mechanics and its Applications, vol. 505, pp. 655665, 2018.

[20] P. Wang and S. Xu, "Spectral coarse grained controllability of complex networks," Physica A: Statistical Mechanics and its Applications, vol. 478, pp. 168-176, 2017.
[21] D. J. Watts and S. H. Strogatz, "Collective dynamics of 'smallworld' networks,” Nature, vol. 393, no. 6684, pp. 440-442, 1998.

[22] N. Dame, "Statistical mechanics of complex networks," Review of Modern Physics, vol. 74, no. 1, pp. 47-97, 2002.

[23] L. Gallos and P. Argyrakis, "Absence of kinetic effects in reaction-diffusion processes in scale-free networks," Physical Review Letters, vol. 92, no. 13, Article ID 138301, 2004.

[24] S. Boccalettia, V. Latorab, Y. Morenod, M. Chavez, and D. Hwang, "Complex networks: structure and dynamics," Physics Reports, vol. 424, no. 4-5, pp. 175-308, 2006.

[25] S. Gómez, A. Díaz-Guilera, J. Gómez-Gardeñes, C. J. PérezVicente, Y. Moreno, and A. Arenas, "Diffusion dynamics on multiplex networks," Physical Review Letters, vol. 110, no. 2, Article ID 028701, 2013.

[26] C. Granell, S. Gómez, and A. Arenas, "Dynamical interplay between awareness and epidemic spreading in multiplex networks," Physical Review Letters, vol. 111, no. 12, Article ID 128701, 2013.

[27] J. Aguirre, R. Sevilla-Escoboza, R. Gutiérrez, D. Papo, and J. M. Buldú, "Synchronization of interconnected networks: the role of connector nodes," Physical Review Letters, vol. 112, no. 24, Article ID 248701, 2014.

[28] M. Xu, J. Lu, and J. Zhou, "Eigenvalue spectrum and synchronization ability of two-layer star networks," Acta Physica Sinica, vol. 65, 2016, in Chinese.

[29] M. Xu, J. Zhou, J. Lu, and X. Wu, "Synchronizability of twolayer networks," The European Physical Journal B, vol. 88, no. 9, p. 240, 2015.

[30] Y. Li, X. Wu, J. Lu et al., "Synchronizability of duplex networks," IEEE Transactions on Circuits and Systems II Express Briefs, vol. 63, no. 2, pp. 206-210, 2017.

[31] J. Sun, X. Li, J. H. Zhang et al., "Synchronizability and eigenvalues of multilayer star networks through unidirectionally coupling," Acta Physica Sinica, vol. 52, p. 188901, 2017, in Chinese.

[32] J. Wei, X. Wu, J.-A. Lu, and X. Wei, "Synchronizability of duplex regular networks," EPL (Europhysics Letters), vol. 120, no. 2, Article ID 20005, 2017.

[33] L. Tang, X. Wu, J. Lü, J. Lu, and R. M. D’Souza, "Master stability functions for complete, intralayer, and interlayer synchronization in multiplex networks of coupled Rössler oscillators," Physical Review E, vol. 99, no. 1, Article ID 012304, 2019.

[34] Y. Deng, Z. Jia, G. Deng, and Q. Zhang, "Eigenvalue spectrum and synchronizability of multiplex chain networks," Physica A: Statistical Mechanics and its Applications, vol. 537, Article ID 122631, 2019. 